\title{
Nonequilibrium Dynamics of Coupled Quantum Systems
}

\author{
G. Flores-Hidalgo ${ }^{a}, *$, Rudnei O. Ramos ${ }^{b}$ \\ a Centro Brasileiro de Pesquisas Fisicas-CBPF, Rua Dr. Xavier Sigaud 150, \\ 22290-180 Rio de Janeiro, RJ, Brazil \\ ${ }^{\mathrm{b}}$ Departamento de Física Teórica, Universidade do Estado do Rio de Janeiro, \\ 20550-013 Rio de Janeiro, RJ, Brazil
}

\begin{abstract}
The nonequilibrium dynamics of coupled quantum oscillators subject to different time dependent quenches are analyzed in the context of the Liouville-von Neumann approach. We consider models of quantum oscillators in interaction that are exactly soluble in the cases of both sudden and smooth quenches. The time evolution of number densities and the final equilibration distribution for the problem of a quantum oscillator coupled to an infinity set of other oscillators (a bath) are explicitly worked out.
\end{abstract}

Key words: Nonequilibrium, thermalization, Liouville-von Neumann PACS: 03.65.Yz, 05.70.Ln, 05.30.Jp

\footnotetext{
* Corresponding author. Email addresses: gflores@cbpf.br (G. Flores-Hidalgo ), rudnei@uerj.br (Rudnei O. Ramos).
} 


\section{Introduction}

The nonequilibrium dynamics of quantum systems are a timely and relevant subject that is important to many areas of study in physical science, from condensed matter systems to cosmology. In most cases we are usually interested in the processes of thermalization and equilibration, the determination of the relevant time scales involved, together with an understanding of the generation of entropy and particle production in nonequilibrium dissipative systems interacting with an environment. However, despite the importance associate to these processes, nonequilibrium problems are still poorly understood (see, e.g. [1]). The nontrivial nonequilibrium dynamics of fields, for instance, have diverse applications, finding use $e . g$. in the studies concerning the recent experiments in ultra-relativistic heavy-ion collision [2]; applications to the current problems of parametric resonance and particle production in cosmology [3]; or in the context of the recent studies involving the intrinsic dissipative nature of interacting fields $[4,5,6]$. In addition to that, typical problems we have in mind to study are those related to the nontrivial out-of-thermal equilibrium dynamics associated with phase transitions in different physical systems. As a few examples we may cite include the current applications to the study of formation of Bose-Einstein condensates after a temperature quench [7], or in the study of the dynamics of coupled fields displaced from their ground states as determined by their free energy densities [8].

One problem that we will be particularly interested in here is that one that involves quenched phase transitions, either caused by a sudden change in temperature of the system, or by a time change of the parameters of the model (like frequency or couplings), in which case we have to deal with situations 
characterized by mixed states involving general density matrices and where an equilibrium or quasi-equilibrium formalism do not apply. Common tools used to study such nonequilibrium quantum systems are the Schwinger's closed time path functional formalism and the canonical quantization methods [9]. In this paper we will mostly be concerned with the understanding of simple, exactly soluble quench scenarios from the stand point of the Liouville-von Neumann (LvN) quantum canonical formalism [10]. In this formalism we have that the time evolution of the density operator $\hat{\rho}$ is given by the $\mathrm{LvN}$ equation with Hamiltonian operator $\hat{H}$ :

$$
\frac{\partial}{\partial t} \hat{\rho}(t)=i[\hat{\rho}(t), \hat{H}]
$$

and satisfying the usual normalization condition $\operatorname{Tr} \hat{\rho}=1$. In order to solve Eq. (1) we can make use of the following fact. Suppose any two operators $\hat{o}_{1}(t)$ and $\hat{o}_{2}(t)$ that satisfy the $\operatorname{LvN}$ equation $(j=1,2)$

$$
\frac{\partial}{\partial t} \hat{o}_{j}(t)=i\left[\hat{o}_{j}(t), \hat{H}\right]
$$

then we can easily show that any operator function of $\hat{o}_{1}(t)$ and $\hat{o}_{2}(t), f\left(\hat{o}_{1}, \hat{o}_{2}\right)$, will also satisfy Eq. (2) provided that the time dependence in $f$ only comes through its dependence in $\hat{o}_{1}$ and $\hat{o}_{2}$. This important property allows us to construct an exact solution to Eq. (1) as a function of time dependent operators that satisfy the LvN equations. The LvN approach has been recently used in Refs. $[11,12]$ to treat different problems in quantum field theory and cosmology and also emphasizing the advantages of the method compared to other approaches for nonequilibrium dynamics of quantum systems. The method may also be more efficient than the more common functional integral method, where most of the time we have to deal with nonlocal terms in time that can 
only be appropriated deal with by recurring to linear response and/or adiabatic approximations (by requiring that the system moves sufficiently slowly as compared to typical microscopic time scales) that may not always apply to physically interesting systems (see for example Refs. $[4,5,6]$ ).

In this paper we will study the use and applicability of the $\mathrm{LvN}$ approach for solving different systems of quantum oscillators subject to different quenches that are exactly soluble. With the study of these systems we expect to gain enough insight to then extend this analysis to more complex problems, including the nontrivial cases involving nonlinear interactions, where usually we must make use of perturbative methods, like in quantum fields in interaction and in the description of system evolution (like through a time dependent order parameter), when interaction with a thermal environment is included. In the next section we will give a simple illustration of use of the LvN approach by solving the (frequency) time dependent harmonic oscillator. Then in Sec. III we will study two examples of interacting quantum systems composed of two harmonic oscillators, initially in different thermal states, that are then coupled linearly at some given time. The case of a smooth quench is explicitly solved. Then in Sec. IV we study the case of a system, represent by an harmonic oscillator, coupled linearly to a bath, represented by an infinity set of other oscillators, like in Caldeira-Leggett models and we explicitly evaluate the number density for the system, in the case of a sudden quench, in the context of the LvN approach. In Sec. V we discuss a general result concerning the addition of nonlinear interactions, for a model with quartic interactions, in the context of the approach discussed in the previous sections. Finally in Sec. VI we give our concluding remarks. Two Appendixes are included to clarify a few technical details. 


\section{The Time Dependent Harmonic Oscillator}

Let us begin by showing how the LvN approach works in the simplest problem of the time dependent harmonic oscillator case, described by the time dependent Hamiltonian:

$$
\hat{H}=\frac{1}{2} \hat{p}^{2}+\frac{1}{2} \omega^{2}(t) \hat{q}^{2},
$$

with a time dependent frequency $\omega(t)$. We consider that initially the system is in thermal equilibrium state at a temperature $T=1 / \beta$ (note that for convenience we use throughout this paper the natural units, $k_{B}=\hbar=1$ ). Next, we introduce time dependent annihilation and creation operators, $\hat{a}(t)$ and $\hat{a}^{\dagger}(t)$, given by

$$
\hat{a}(t)=A(t) \hat{q}+B(t) \hat{p}
$$

and

$$
\hat{a}^{\dagger}(t)=A^{*}(t) \hat{q}+B^{*}(t) \hat{p},
$$

where the time dependence comes through the c-number coefficients $A(t)$ and $B(t)$. From the relation

$$
\left[\hat{a}(t), \hat{a}^{\dagger}(t)\right]=1,
$$

we obtain that

$$
A(t) B^{*}(t)-B(t) A^{*}(t)=-i .
$$


Using Eq. (4) in Eq. (2), we obtain

$$
\dot{A}(t) \hat{q}+\dot{B}(t) \hat{p}=-A(t) \hat{p}+\omega^{2}(t) B(t) \hat{q},
$$

where dots mean time derivatives. From Eq. (8) we then get that

$$
\ddot{B}(t)+\omega^{2}(t) B(t)=0
$$

and $A(t)$ is related to $B(t)$ by the relation

$$
A(t)=-\dot{B}(t) .
$$

Using the above equation we can rewrite Eqs. (4), (5) and (7) and express them as

$$
\begin{aligned}
& \hat{a}(t)=B(t) \hat{p}-\dot{B}(t) \hat{q}, \\
& \hat{a}^{\dagger}(t)=B^{*}(t) \hat{p}-\dot{B}^{*}(t) \hat{q}
\end{aligned}
$$

and

$$
\dot{B}(t) B^{*}(t)-B(t) \dot{B}^{*}(t)=i .
$$

It is easy to see that Eq. (13) is the Wronskian of Eq. (9), i.e., warranting that the operators $\hat{a}(t)$ and $\hat{a}^{\dagger}(t)$ are annihilation and creations operators at all times. Now since our annihilation and creation operators satisfy the LvN equation, any function of them must also equally satisfy it too. In particular we can construct an exact solution for $\hat{\rho}(t)$. Since our initial condition is one of thermal equilibrium we can, therefore, write

$$
\hat{\rho}(t)=\frac{1}{Z_{\beta}} \exp \left\{-\beta \omega_{0}\left[\hat{a}^{\dagger}(t) \hat{a}(t)+\frac{1}{2}\right]\right\},
$$


where

$$
Z_{\beta}=\operatorname{Tr} \exp \left\{-\beta \omega_{0}\left[\hat{a}^{\dagger}(t) \hat{a}(t)+\frac{1}{2}\right]\right\}
$$

and $\omega_{0}=\omega(t \rightarrow-\infty)$, i.e, we are choosing as initial condition that our system is at thermal equilibrium at $t \rightarrow-\infty$ with temperature $1 / \beta$. Since relation (6) is satisfied, we can define a time dependent number operator $\hat{N}(t)=\hat{a}^{\dagger}(t) \hat{a}(t)$, with time dependent number eigenvectors $|n(t)\rangle$. However, because Eq. (6) is a time independent relation, the number eigenvalues $n$ are time independent, i.e, we have

$$
\hat{N}(t)|n(t)\rangle=n|n(t)\rangle \text {. }
$$

Using Eq. (16) it is easy to show that $Z_{\beta}$ is the partition function for the initial thermal equilibrium state,

$$
\begin{aligned}
Z_{\beta} & =\sum_{n=0}^{\infty}\left\langle n(t)\left|\exp \left\{-\beta \omega_{0}\left[\hat{N}(t)+\frac{1}{2}\right]\right\}\right| n(t)\right\rangle \\
& =\sum_{n=0}^{\infty} \exp \left\{-\beta \omega_{0}\left(n+\frac{1}{2}\right)\right\} \\
& =\frac{1}{2 \sinh \left(\frac{\beta \omega_{0}}{2}\right)}
\end{aligned}
$$

Note that $\hat{\rho}(t)$, given by Eq. (14), solves exactly the out-of-equilibrium dynamics of the system with the condition of thermal equilibrium as initial state. In order to compute the time dependent thermal averages of certain operators it is convenient to rewrite Eqs. (11) and (12) in terms of the initial annihilation and creation operators, that we will denote simple as $\hat{a}$ and $\hat{a}^{\dagger}$. For this end we first solve for $\hat{q}$ and $\hat{p}$ in terms of the initial values of $B(t)$ and $\dot{B}(t)$ that we will denote just as $B$ and $\dot{B}$, respectively, without the time argument. We 
then find that

$$
\begin{aligned}
& \hat{q}=i\left(B^{*} \hat{a}-B \hat{a}^{\dagger}\right), \\
& \hat{p}=i\left(\dot{B}^{*} \hat{a}-\dot{B} \hat{a}^{\dagger}\right) .
\end{aligned}
$$

Using now Eqs. (18) and (19) in Eqs. (11) and (12), we then obtain the Bogoliubov transformation:

$$
\left(\begin{array}{c}
\hat{a}(t) \\
\hat{a}^{\dagger}(t)
\end{array}\right)=\left(\begin{array}{cc}
\alpha(t) & \beta(t) \\
\beta^{*}(t) & \alpha^{*}(t)
\end{array}\right)\left(\begin{array}{c}
\hat{a} \\
\hat{a}^{\dagger}
\end{array}\right)
$$

where $\alpha(t)$ and $\beta(t)$, the Bogoliubov coefficients, are given by

$$
\begin{aligned}
& \alpha(t)=i\left(\dot{B}^{*} B(t)-B^{*} \dot{B}(t)\right), \\
& \beta(t)=i(B \dot{B}(t)-\dot{B} B(t)) .
\end{aligned}
$$

In terms of $\alpha(t)$ and $\beta(t)$ Eq. (13) gives then the following relation:

$$
|\alpha(t)|^{2}-|\beta(t)|^{2}=1
$$

The utility of Eq. (20) is clear once we compute the thermal expectation value of the number operator $\hat{N}$,

$$
\begin{aligned}
\bar{n}(t) & =\operatorname{Tr}\left[\hat{\rho}(t) \hat{a}^{\dagger} \hat{a}\right] \\
& =\frac{1}{Z_{\beta}} \sum_{n=0}^{\infty}\left\langle n(t)\left|\exp \left\{-\beta \omega_{0}\left[\hat{a}^{\dagger}(t) \hat{a}(t)+\frac{1}{2}\right]\right\} \hat{a}^{\dagger} \hat{a}\right| n(t)\right\rangle .
\end{aligned}
$$


Solving Eq. (20) for $\hat{a}$ and $\hat{a}^{\dagger}$ in terms of $\hat{a}(t)$ and $\hat{a}^{\dagger}(t)$, substituting them in Eq. (24) and using Eq. (16), it is easy to find that

$$
\bar{n}(t)=\left[|\alpha(t)|^{2}+|\beta(t)|^{2}\right] \bar{n}+|\beta(t)|^{2},
$$

where

$$
\bar{n}=\frac{1}{e^{\beta \omega_{0}}-1}
$$

is the initial thermal equilibrium average for the number operator.

\subsection{The Time Independent Harmonic Oscillator}

Let us first consider the trivial case of the time independent oscillator, with $\omega^{2}(t)=\omega_{0}^{2}$ as an example of application. In this case the solution for Eq. (9) is just

$$
B(t)=a e^{i \omega_{0} t}+b e^{-i \omega_{0} t}
$$

substituting the above expression for $B(t)$ in Eq. (13) we obtain

$$
|a|^{2}-|b|^{2}=\frac{1}{2 \omega_{0}}
$$

In this case we can obtain a time independent number operator choosing $b=0$ and $|a|=1 / \sqrt{2 \omega_{0}}$. The other choice $a=0$ and $|b| \neq 0$ is simply ruled out since in this case we would get $|b|^{2}<0$. Choosing a null phase for $a$, we then obtain the solutions

$$
\hat{a}(t)=\frac{e^{i \omega_{0} t}}{\sqrt{2 \omega_{0}}}\left(\hat{p}-i \omega_{0} \hat{q}\right)
$$


and

$$
\hat{a}^{\dagger}(t)=\frac{e^{-i \omega_{0} t}}{\sqrt{2 \omega_{0}}}\left(\hat{p}+i \omega_{0} \hat{q}\right) .
$$

Although the creation and annihilation operators are time dependent the number operator $\hat{N}=\hat{a}^{\dagger}(t) \hat{a}(t)$ is time independent, as expected in this trivial example.

\subsection{An Exactly Soluble Time Dependent Harmonic Oscillator}

Let us consider now the case of an harmonic oscillator with the time dependent frequency:

$$
\omega^{2}(t)=\frac{\omega^{2}}{\cosh ^{2}(t / \tau)}+\omega_{0}^{2} .
$$

Here the function $B(t)$ satisfies the differential equation

$$
\ddot{B}(t)+\left[\frac{\omega^{2}}{\cosh ^{2}(t / \tau)}+\omega_{0}^{2}\right] B(t)=0
$$

Eq. (32) can be solved with the condition that at $t \rightarrow-\infty$ the system is at thermal equilibrium at temperature $T$. From the results of the previous subsection we then have to solve Eq. (32) with the asymptotic condition that

$$
B(t \rightarrow-\infty)=\frac{e^{i \omega_{0} t}}{\sqrt{2 \omega_{0}}}
$$

The above problem is formally identical as an one-dimensional quantum scattering problem. The solution of Eq. (32), under the asymptotic condition given by Eq. (33) is [13]

$$
B(t)=\frac{2^{-i \tau \omega_{0}}}{\sqrt{2 \omega_{0}}}[\operatorname{sech}(t / \tau)]^{i \tau \omega_{0}} F\left[\tilde{a}, \tilde{b}, \tilde{c}, \frac{1}{2}+\frac{1}{2} \tanh (t / \tau)\right]
$$


where $F(x, y, z, w)$ is the Gauss' hypergeometric function [14] and the coefficients $\tilde{a}, \tilde{b}$ and $\tilde{c}$ are given by

$$
\begin{aligned}
& \tilde{a}=\frac{1}{2}+i \omega_{0} \tau+\sqrt{\omega^{2} \tau^{2}+\frac{1}{4}}, \\
& \tilde{b}=\frac{1}{2}+i \omega_{0} \tau-\sqrt{\omega^{2} \tau^{2}+\frac{1}{4}}
\end{aligned}
$$

and

$$
\tilde{c}=1+i \omega_{0} \tau \text {. }
$$

When $t \rightarrow \infty$ intuitively one expects that the system will thermalize. Taking the $t \rightarrow \infty$ limit in Eq. (34) we obtain the result

$$
B(t \rightarrow \infty)=\frac{\Gamma(\tilde{c}) \Gamma(\tilde{a}+\tilde{b}-\tilde{c})}{\Gamma(\tilde{a}) \Gamma(\tilde{b})} \frac{e^{i \omega_{0} t}}{\sqrt{2 \omega_{0}}}+\frac{\Gamma(\tilde{c}) \Gamma(\tilde{c}-\tilde{a}-\tilde{b})}{\Gamma(\tilde{c}-\tilde{a}) \Gamma(\tilde{c}-\tilde{b})} \frac{e^{-i \omega_{0} t}}{\sqrt{2 \omega_{0}}}
$$

Substituting Eq. (38) in Eqs. (21) and (22) we obtain for $\bar{n}(t)$, given by Eq. (25), an expression with oscillatory behavior in general. For these cases clearly the system never reaches thermal equilibrium. But if we analyze with some care the arguments of the gamma-functions in Eq. (38), we see that for those values of $\tau$ such that $\tau^{2} \omega^{2}=N(N+1)$, with $N$ integer, then the second term in Eq. (38) vanishes. In this case the system reaches a final thermal equilibrium state with final temperature that can be shown to be the same as of the initial one. This same result was also obtained previously in Ref. [15] whose authors have studied this problem solving the $\mathrm{LvN}$ equation obtained from a variational principle. We can also show that in the final thermal equilibrium state there will be no dependences on parameters other than those of the initial thermal equilibrium state $\left(\omega_{0}\right.$ and $\left.\beta\right)$. All the factors that appear multiplying the exponential factor in the first term in Eq. (38) contribute 
with a time independent phase factor. This is equivalent to what happens in the one dimensional scattering problem as consequence of the conservation of probability in a reflectionless potential [13]. In computing physical quantities the phase factor cancels out and then, when the system reaches a final thermal equilibrium state it loses all the information about how this state was reached. Implicitly we have here a manifestation of decoherence already at the level of this simple problem as a consequence of a particular quench scenario and that will be so important when models with larger degrees of freedom are taken into account.

As an example of a concrete result, let us consider for convenience the case $N=1$, i.e, when $\tau^{2} \omega^{2}=2$. In this case $B(t)$ reduces to

$$
B(t)=\frac{e^{i \omega_{0} t}}{\sqrt{2 \omega_{0}}\left(1+i \omega_{0} \tau\right)}\left[i \omega_{0} \tau-\tanh (t / \tau)\right]
$$

Substituting Eq. (39) in Eqs. (21) and (22) we get the results

$$
|\alpha(t)|^{2}=1+\frac{\left[1-\tanh ^{2}(t / \tau)\right]^{2}}{4 \omega_{0}^{2} \tau^{2}\left(1+\omega_{0}^{2} \tau^{2}\right)}
$$

and

$$
|\beta(t)|^{2}=\frac{\left[1-\tanh ^{2}(t / \tau)\right]^{2}}{4 \omega_{0}^{2} \tau^{2}\left(1+\omega_{0}^{2} \tau^{2}\right)}
$$

Using these equations for $|\alpha(t)|^{2}$ and $|\beta(t)|^{2}$ in Eq. (25) we then obtain for $\bar{n}(t)$ the result:

$$
\bar{n}(t)=\left(1+\frac{\left[1-\tanh ^{2}(t / \tau)\right]^{2}}{2 \omega_{0}^{2} \tau^{2}\left(1+\omega_{0}^{2} \tau^{2}\right)}\right) \bar{n}+\frac{\left[1-\tanh ^{2}(t / \tau)\right]^{2}}{4 \omega_{0}^{2} \tau^{2}\left(1+\omega_{0}^{2} \tau^{2}\right)}
$$


Results for $\bar{n}(t)$ for $\omega_{0} \beta=1$ and for different values of the "decay time" $\tau$ are shown in Fig. 1. Note that the peak at $t=0$ just represents the point around which the time-dependent frequency $\omega(t)$, Eq. (31), reaches its maximum value and the peak is higher the smaller is the decay time, in agreement to the fact that, from Eq. (41), the smallest is $\tau$ the largest is the production of excited states (particles, in the context of quantum fields), as represented by $|\beta(t)|^{2}$.

\section{Time Dependent Quantum Interacting Oscillators}

Lets us now consider a system of two time dependent oscillators, linearly coupled with interaction strength $\lambda(t)$, with Hamiltonian given by

$$
\hat{H}=\frac{1}{2} \sum_{j=1}^{2}\left[\hat{p}_{j}^{2}+\omega_{j}^{2}(t) \hat{q}_{j}^{2}\right]+\lambda(t) \hat{q}_{1} \hat{q}_{2} .
$$

The time dependent annihilation and creation operators can now be written as $(i=1,2)$

$$
\hat{a}_{i}(t)=\sum_{j=1}^{2}\left[B_{i j}(t) \hat{p}_{j}-\dot{B}_{i j}(t) \hat{q}_{j}\right]
$$

and

$$
\hat{a}_{i}^{\dagger}(t)=\sum_{j=1}^{2}\left[B_{i j}^{*}(t) \hat{p}_{j}-\dot{B}_{i j}^{*}(t) \hat{q}_{j}\right],
$$

In an identical way as we have performed in the single time dependent oscillator of last section, we require that the operators $\hat{a}_{i}(t)$ and $\hat{a}_{i}^{\dagger}(t)$ satisfy the LvN equation, Eq. (2). From this requirement the following equations for the time dependent coefficients $B_{i j}(t)$ follow:

$$
\ddot{B}_{i 1}(t)+\omega_{1}^{2}(t) B_{i 1}(t)+\lambda(t) B_{i 2}(t)=0
$$


and

$$
\ddot{B}_{i 2}(t)+\omega_{2}^{2}(t) B_{i 2}(t)+\lambda(t) B_{i 1}(t)=0
$$

The condition

$$
\left[\hat{a}_{i}(t), \hat{a}_{j}^{\dagger}(t)\right]=\delta_{i j}
$$

leads now to the equation

$$
\sum_{k=1}^{2}\left[\dot{B}_{i k}(t) B_{j k}^{*}(t)-B_{i k}(t) \dot{B}_{j k}^{*}(t)\right]=i \delta_{i j}
$$

and the identities

$$
\left[\hat{a}_{i}(t), \hat{a}_{j}(t)\right]=\left[\hat{a}_{i}^{\dagger}(t), \hat{a}_{j}^{\dagger}(t)\right]=0
$$

lead, respectively, to the following additional equations for $B_{i j}(t)$ :

$$
\begin{aligned}
& \sum_{k=1}^{2}\left[B_{i k}(t) \dot{B}_{j k}(t)-\dot{B}_{i k}(t) B_{j k}(t)\right]=0 \\
& \sum_{k=1}^{2}\left[B_{i k}^{*}(t) \dot{B}_{j k}^{*}(t)-\dot{B}_{i k}^{*}(t) B_{j k}^{*}(t)\right]=0
\end{aligned}
$$

Using Eqs. (46) and (47), it can be shown that Eqs. (49), (50) and (51) must be time independent. As in the one single harmonic oscillator case we can write the time dependent annihilation and creation operators as functions of the initial annihilation and creation operators (that we just call by $\hat{a}_{i}$ and $\hat{a}_{i}^{\dagger}$, respectively, without the time argument, as indicating that these quantities should be evaluated at the initial time). This can be accomplished by using the requirement that initially the system consists of two independent harmonic 
oscillators with frequencies $\omega_{1}$ and $\omega_{2}$, each one at well defined thermal equilibrium states with temperatures $1 / \beta_{1}$ and $1 / \beta_{2}$, respectively. In this case the matrix $\{B\}$, at the initial time, has elements $B_{i j}$ given by

$$
B_{i j}=\delta_{i j} \frac{e^{i \omega_{i} t}}{\sqrt{2 \omega_{i}}} .
$$

We can now readily show that the Bogoliubov transformation in this case is given by

$$
\left(\begin{array}{c}
\hat{a}_{i}(t) \\
\hat{a}_{i}^{\dagger}(t)
\end{array}\right)=\sum_{j=1}^{2}\left(\begin{array}{cc}
\alpha_{i j}(t) & \beta_{i j}(t) \\
\beta_{i j}^{*}(t) & \alpha_{i j}^{*}(t)
\end{array}\right)\left(\begin{array}{c}
\hat{a}_{j} \\
\hat{a}_{j}^{\dagger}
\end{array}\right),
$$

where

$$
\alpha_{i j}(t)=i\left(\dot{B}_{j j}^{*} B_{i j}(t)-B_{j j}^{*} \dot{B}_{i j}(t)\right)
$$

and

$$
\beta_{i j}(t)=i\left(B_{j j} \dot{B}_{i j}(t)-\dot{B}_{j j} B_{i j}(t)\right)
$$

In terms of the above Bogoliubov coefficients the conditions (49) and (51) are given, respectively, by

$$
\sum_{k=1}^{2}\left[\alpha_{i k}(t) \alpha_{j k}^{*}(t)-\beta_{i k}(t) \beta_{j k}^{*}(t)\right]=\delta_{i j}
$$

and

$$
\begin{aligned}
& \sum_{k=1}^{2}\left[\alpha_{i k}(t) \beta_{j k}(t)-\beta_{i k}(t) \alpha_{j k}(t)\right]=0, \\
& \sum_{k=1}^{2}\left[\alpha_{i k}^{*}(t) \beta_{j k}^{*}(t)-\beta_{i k}^{*}(t) \alpha_{j k}^{*}(t)\right]=0 .
\end{aligned}
$$


Since $\hat{a}_{i}(t)$ and $\hat{a}_{i}^{\dagger}(t)$ are solutions of the LvN equation, the exact solution for the time dependent density operator with thermal equilibrium initial conditions is

$$
\hat{\rho}(t)=\frac{1}{Z_{\beta_{1}} Z_{\beta_{2}}} \exp \left\{-\sum_{i=1}^{2} \beta_{i} \omega_{i}\left[\hat{a}_{i}^{\dagger}(t) \hat{a}_{i}(t)+\frac{1}{2}\right]\right\},
$$

where $Z_{\beta_{i}}, i=1,2$, are the initial (equilibrium) partition functions for each oscillator as given by Eq. (17). Note that the out-of-equilibrium problem resulting from the coupling of the two systems at an initial time $t_{0}$ is reduced to solving the two coupled second order differential equations given by Eqs. (46) and (47). As in the time dependent single harmonic oscillator case we can compute the time dependent thermal expectation values for the number operators, $\hat{N}_{i}=\hat{a}_{i}^{\dagger} \hat{a}_{i}$

$$
\begin{aligned}
& \bar{n}_{i}(t)= \operatorname{Tr}\left[\hat{\rho}(t) \hat{a}_{i}^{\dagger} \hat{a}_{i}\right] \\
&=\frac{1}{Z_{\beta_{1}} Z_{\beta_{2}}} \sum_{n_{1}, n_{2}=0}^{\infty}\left\langle n_{1}(t), n_{2}(t)\right| \exp \left\{-\sum_{j=1}^{2} \beta_{j} \omega_{j}\left[\hat{a}_{j}^{\dagger}(t) \hat{a}_{j}(t)+\frac{1}{2}\right]\right\} \\
& \hat{a}_{i}^{\dagger} \hat{a}_{i}\left|n_{1}(t), n_{2}(t)\right\rangle .
\end{aligned}
$$

In order to evaluate the above expression we have to solve Eq. (53) for $\hat{a}_{i}$ and $\hat{a}_{j}^{\dagger}$ in terms of $\hat{a}_{i}(t), \hat{a}_{j}^{\dagger}(t)$. This can be easily done using Eqs. (56) and (57) then obtaining

$$
\left(\begin{array}{c}
\hat{a}_{i} \\
\hat{a}_{i}^{\dagger}
\end{array}\right)=\sum_{j=1}^{2}\left(\begin{array}{cc}
\alpha_{i j}^{*}(t) & -\beta_{j i}(t) \\
-\beta_{j i}^{*}(t) & \alpha_{i j}(t)
\end{array}\right)\left(\begin{array}{c}
\hat{a}_{j}(t) \\
\hat{a}_{j}^{\dagger}(t)
\end{array}\right) .
$$

Using Eq. (60) in Eq. (59) we obtain

$$
\bar{n}_{i}(t)=\sum_{j=1}^{2}\left[\left(\left|\alpha_{i j}(t)\right|^{2}+\left|\beta_{j i}(t)\right|^{2}\right) \bar{n}_{j}+\left|\beta_{j i}(t)\right|^{2}\right],
$$


which generalizes Eq. (25) for the case of more than one quantum oscillator. In Eq. (61) $\bar{n}_{j}$ is the initial thermal equilibrium distribution,

$$
\bar{n}_{j}=\frac{1}{e^{\beta_{j} \omega_{j}}-1} .
$$

\subsection{Solution for the Instantaneous Interaction}

As a first application of the above equations, let us consider the example of two coupled quantum oscillators with time independent frequencies and coupling $\lambda(t)$ suddenly turned on at time $t=0$, i.e,

$$
\lambda(t)=\left\{\begin{array}{c}
0, t \leq 0 \\
\lambda, t>0
\end{array} .\right.
$$

In this case we have for $t \leq 0$ that

$$
B_{i j}(t \leq 0)=\left(\begin{array}{cc}
\frac{e^{i \omega_{1} t}}{\sqrt{2 \omega_{1}}} & 0 \\
0 & \frac{e^{i \omega_{2} t}}{\sqrt{2 \omega_{2}}}
\end{array}\right)
$$

and for $t>0$ we can solve Eqs. (46) and (47) in terms of normal coordinates $Q_{i j}(t)$, defined in terms of $B_{i j}(t)$ by

$$
B_{i j}(t)=\sum_{k=1}^{2} \eta_{j k} Q_{i k}(t),
$$

where the matrix transformation $\eta_{i j}$ is time independent. Substituting the above equation in Eqs. (46) and (47) we obtain (for details see appendix A)

$$
\ddot{Q}_{i k}(t)+\Omega_{k}^{2} Q_{i k}(t)=0,
$$


provided that $\eta_{i j}$ is an orthogonal matrix with elements given by

$$
\begin{aligned}
& \eta_{1 j}=\left[1+\frac{\lambda^{2}}{\left(\omega_{2}^{2}-\Omega_{j}^{2}\right)^{2}}\right]^{-\frac{1}{2}} \\
& \eta_{2 j}=\frac{\lambda}{\Omega_{j}^{2}-\omega_{2}^{2}} \eta_{1 j}
\end{aligned}
$$

and $\Omega_{j}$ are obtained as the solutions of

$$
\left(\omega_{1}^{2}-\Omega_{j}^{2}\right)\left(\omega_{2}^{2}-\Omega_{j}^{2}\right)=\lambda^{2}
$$

from which we obtain

$$
\begin{aligned}
& \Omega_{1}^{2}=\frac{1}{2}\left[\omega_{1}^{2}+\omega_{2}^{2}+\sqrt{\left(\omega_{1}^{2}-\omega_{2}^{2}\right)^{2}+4 \lambda^{2}}\right], \\
& \Omega_{2}^{2}=\frac{1}{2}\left[\omega_{1}^{2}+\omega_{2}^{2}-\sqrt{\left(\omega_{1}^{2}-\omega_{2}^{2}\right)^{2}+4 \lambda^{2}}\right] .
\end{aligned}
$$

Solving Eq. (66) and substituting it in Eq. (65), we obtain for $B_{i j}(t)$ the following expression

$$
B_{i j}(t)=\sum_{k=1}^{2} \eta_{j k}\left(a_{i k} e^{i \Omega_{k} t}+b_{i k} e^{-i \Omega_{k} t}\right)
$$

where $a_{i k}$ and $b_{i k}$ are constants of integration to be determined by the initial conditions. Since at $t=0, B_{i j}(t)$ and $\dot{B}_{i j}(t)$ are continuous, then from Eqs. (64) and (71), we obtain that the coefficients $a_{i k}$ and $b_{i k}$, appearing in Eq. (71), are given by

$$
\begin{aligned}
& a_{i k}=\frac{\eta_{i k}}{\sqrt{8 \omega_{i}}}\left(1+\frac{\omega_{i}}{\Omega_{k}}\right), \\
& b_{i k}=\frac{\eta_{i k}}{\sqrt{8 \omega_{i}}}\left(1-\frac{\omega_{i}}{\Omega_{k}}\right) .
\end{aligned}
$$


Substituting Eq. (72) back in Eq. (71), we obtain the result

$$
B_{i j}(t)=\sum_{k=1}^{2} \frac{\eta_{i k} \eta_{j k}}{\sqrt{2 \omega_{i}}}\left[\cos \left(\Omega_{k} t\right)+\frac{i \omega_{i}}{\Omega_{k}} \sin \left(\Omega_{k} t\right)\right]
$$

Using Eq. (64) for $B_{i j}$ at $t=0$ in Eq. (54) we obtain for the Bogoliubov coefficients the expressions

$$
\alpha_{i j}(t)=\sqrt{\frac{\omega_{j}}{2}} B_{i j}(t)-\frac{i}{\sqrt{2 \omega_{j}}} \dot{B}_{i j}(t)
$$

and

$$
\beta_{i j}(t)=\sqrt{\frac{\omega_{j}}{2}} B_{i j}(t)+\frac{i}{\sqrt{2 \omega_{j}}} \dot{B}_{i j}(t)
$$

Eqs. (73) - (75) together with Eqs. (67) - (70) completely solve the out-ofequilibrium evolution of the system in consideration. In this case the system never reaches a final thermal equilibrium state since the Bogoliubov coefficients obtained using the result for $B_{i j}(t)$, as given by Eq. (73), have an oscillatory behavior for all $t$. The solutions for $\bar{n}_{1}(t)$ and $\bar{n}_{2}(t)$ are shown in Fig. 2 for the case of $\omega_{1}=\omega_{2}$. This will be further discussed below.

\subsection{An Exactly Soluble Smooth Quench}

As an example of non-instantaneous quench and exactly soluble model, let us consider next the case of a smooth quench with the linear coupling given by the expression

$$
\lambda(t)=\frac{\lambda}{2}[1+\tanh (t / \tau)],
$$


and with time independent frequencies $\omega_{1}=\omega_{2}=\omega$. The equations for the $B_{i j}(t)$ functions now become

$$
\ddot{B}_{i 1}(t)+\omega^{2} B_{i 1}(t)+\frac{\lambda}{2}[1+\tanh (t / \tau)] B_{i 2}(t)=0
$$

and

$$
\ddot{B}_{i 2}(t)+\omega^{2} B_{i 2}(t)+\frac{\lambda}{2}[1+\tanh (t / \tau)] B_{i 1}(t)=0 .
$$

Eqs. (77) and (78) can be more easily solved if we make use of the following transformation:

$$
\begin{aligned}
R_{i}(t) & =B_{i 1}(t)-B_{i 2}(t) \\
G_{i}(t) & =B_{i 1}(t)+B_{i 2}(t),
\end{aligned}
$$

which then lead to the following uncoupled differential equations for $R_{i}(t)$ and $G_{i}(t)$

$$
\ddot{R}_{i}(t)+\left\{\omega^{2}-\frac{\lambda}{2}[1+\tanh (t / \tau)]\right\} R_{i}(t)=0
$$

and

$$
\ddot{G}_{i}(t)+\left\{\omega^{2}+\frac{\lambda}{2}[1+\tanh (t / \tau)]\right\} G_{i}(t)=0 .
$$

Note that even for the case of time dependent frequencies $\omega_{i}(t)$, the Eqs. $(77)$ and (78) still uncouple and can be put in the form of Eqs. (80) and (81). The solutions for Eqs. (80) and (81) can now be found to be given by

$$
R_{i}(t)=A_{i} e^{-i \omega t} F\left[-a_{1},-b_{1}, c^{*},-e^{2 t / \tau}\right]+B_{i} e^{i \omega t} F\left[b_{1}, a_{1}, c,-e^{2 t / \tau}\right]
$$


and

$$
G_{i}(t)=C_{i} e^{-i \omega t} F\left[-a_{2},-b_{2}, c^{*},-e^{2 t / \tau}\right]+D_{i} e^{i \omega t} F\left[b_{2}, a_{2}, c,-e^{2 t / \tau}\right]
$$

where

$$
\begin{aligned}
& a_{1}=\frac{i \tau}{2}\left(\omega-\Omega_{2}\right), \\
& a_{2}=\frac{i \tau}{2}\left(\omega-\Omega_{1}\right), \\
& b_{1}=\frac{i \tau}{2}\left(\omega+\Omega_{2}\right), \\
& b_{2}=\frac{i \tau}{2}\left(\omega+\Omega_{1}\right), \\
& c=1+i \omega \tau,
\end{aligned}
$$

and $\Omega_{1(2)}=\sqrt{\omega^{2} \pm|\lambda|}$. We assume that $\omega^{2}>|\lambda|$ in order to avoid runaway solutions. Inverting the transformation given by (79) we now obtain that

$$
\begin{aligned}
B_{i 1}=\frac{1}{2} & \left\{C_{i} e^{-i \omega t} F\left[-a_{2},-b_{2}, c^{*},-e^{2 t / \tau}\right]+D_{i} e^{i \omega t} F\left[b_{2}, a_{2}, c,-e^{2 t / \tau}\right]\right. \\
& \left.+A_{i} e^{-i \omega t} F\left[-a_{1},-b_{1}, c^{*},-e^{2 t / \tau}\right]+B_{i} e^{i \omega t} F\left[b_{1}, a_{1}, c,-e^{2 t / \tau}\right]\right\},
\end{aligned}
$$

and

$$
\begin{aligned}
B_{i 2}=\frac{1}{2} & \left\{C_{i} e^{-i \omega t} F\left[-a_{2},-b_{2}, c^{*},-e^{2 t / \tau}\right]+D_{i} e^{i \omega t} F\left[b_{2}, a_{2}, c,-e^{2 t / \tau}\right]\right. \\
& \left.-A_{i} e^{-i \omega t} F\left[-a_{1},-b_{1}, c^{*},-e^{2 t / \tau}\right]-B_{i} e^{i \omega t} F\left[b_{1}, a_{1}, c,-e^{2 t / \tau}\right]\right\},
\end{aligned}
$$

Using the initial conditions (at $t \rightarrow-\infty)$,

$$
\begin{aligned}
& B_{11}=B_{22}=\frac{\exp (i \omega t)}{\sqrt{2 \omega}} \\
& B_{12}=B_{21}=0
\end{aligned}
$$

we obtain the constants $A_{i}, B_{i}, C_{i}$ and $D_{i}$ as given by: $A_{1}=A_{2}=C_{1}=C_{2}=0$ and $B_{1}=-B_{2}=D_{1}=D_{2}=1 / \sqrt{2 \omega}$. Then, finally, we find the solutions for 
the time dependent factors $B_{i j}(t)$ as given by the expressions

$$
\begin{aligned}
& B_{11}(t)=B_{22}(t)=\frac{e^{i \omega t}}{2 \sqrt{2 \omega}}\left\{F\left[b_{1}, a_{1}, c,-e^{2 t / \tau}\right]+F\left[b_{2}, a_{2}, c,-e^{2 t / \tau}\right]\right\}, \\
& B_{12}(t)=B_{21}(t)=\frac{e^{i \omega t}}{2 \sqrt{2 \omega}}\left\{F\left[b_{1}, a_{1}, c,-e^{2 t / \tau}\right]-F\left[b_{2}, a_{2}, c,-e^{2 t / \tau}\right]\right\} .
\end{aligned}
$$

The asymptotic behavior $(t \gg \tau)$ for the functions $B_{i j}(t)$ follows from the asymptotic behavior of the hypergeometric functions [16], which leads to the results

$$
\begin{aligned}
B_{11}(t \rightarrow \infty)=\frac{1}{\sqrt{8 \omega_{0}}} & {\left[\frac{\Gamma(c) \Gamma\left(a_{1}-b_{1}\right)}{\Gamma\left(a_{1}\right) \Gamma\left(c-b_{1}\right)} e^{i \Omega_{2} t}+\frac{\Gamma(c) \Gamma\left(b_{1}-a_{1}\right)}{\Gamma\left(b_{1}\right) \Gamma\left(c-a_{1}\right)} e^{-i \Omega_{2} t}\right.} \\
+ & \left.\frac{\Gamma(c) \Gamma\left(a_{2}-b_{2}\right)}{\Gamma\left(a_{2}\right) \Gamma\left(c-b_{2}\right)} e^{i \Omega_{1} t}+\frac{\Gamma(c) \Gamma\left(b_{2}-a_{2}\right)}{\Gamma\left(b_{2}\right) \Gamma\left(c-a_{2}\right)} e^{-i \Omega_{1} t}\right]
\end{aligned}
$$

and

$$
\begin{aligned}
B_{12}(t \rightarrow \infty)=\frac{1}{\sqrt{8 \omega_{0}}} & {\left[\frac{\Gamma(c) \Gamma\left(a_{1}-b_{1}\right)}{\Gamma\left(a_{1}\right) \Gamma\left(c-b_{1}\right)} e^{i \Omega_{2} t}+\frac{\Gamma(c) \Gamma\left(b_{1}-a_{1}\right)}{\Gamma\left(b_{1}\right) \Gamma\left(c-a_{1}\right)} e^{-i \Omega_{2} t}\right.} \\
& \left.-\frac{\Gamma(c) \Gamma\left(a_{2}-b_{2}\right)}{\Gamma\left(a_{2}\right) \Gamma\left(c-b_{2}\right)} e^{i \Omega_{1} t}-\frac{\Gamma(c) \Gamma\left(b_{2}-a_{2}\right)}{\Gamma\left(b_{2}\right) \Gamma\left(c-a_{2}\right)} e^{-i \Omega_{1} t}\right] .
\end{aligned}
$$

Note that Eqs. (89) and (90) are similar to Eq. (71) for the case of an instantaneous quench and with $\omega_{1}=\omega_{2}$. Therefore we expect that both cases will lead to qualitative similar results in the asymptotic limit with both oscillators never reaching a final equilibrium state. This is a consequence that in both cases the models considered are purely reversible systems and therefore energy is transferred in a reversible way from one oscillator to the other, with the system never actually equilibrating or thermalizing. This situation is changed considerably when the system is coupled to an environment, modeled for instance as infinity other oscillators coupled to one given oscillator, just like in the Caldeira-Leggett type of models [17]. Under this situation energy 
can now be transferred irreversibly from the system of oscillators to the environment leading eventually to equilibration and thermalization of the system. How these processes come about will be explicitly shown in the next section in the context of the LvN approach.

\section{A system in interaction with a bath: an oscillator interacting with $N \rightarrow \infty$ other oscillators}

In this section we consider a system composed by an oscillator interacting linearly with $N \rightarrow \infty$ other oscillators (here to be considered as the environment or bath, in the typical denomination of Caldeira-Leggett type of models [17]). The Hamiltonian is now described by

$$
\hat{H}=\frac{1}{2} \hat{p}_{0}^{2}+\frac{\omega_{0}^{2}}{2} \hat{q}_{0}^{2}+\frac{1}{2} \sum_{k=1}^{N}\left[\hat{p}_{k}^{2}+\omega_{k}^{2} \hat{q}_{k}^{2}+2 \lambda_{k}(t) \hat{q}_{0} \hat{q}_{k}\right]
$$

In the above $\lambda_{k}(t)$ is a time dependent coupling constant (with dimension of the square of a frequency). In general the frequencies can also be made time dependent, but this case will not be explicitly considered here.

We consider that initially the oscillator and the environment are each sepa-

rately at thermal equilibrium states at inverse temperatures $\beta_{0}$ and $\beta$, respectively (we do not concern here about how this initial state can be realized physically). That is, we consider that initially $\lambda_{k}(t)=0$. Then the initial density operator $\hat{\rho}_{0}$, of the total system will be given by

$$
\hat{\rho}_{0}=\hat{\rho}_{\beta_{0}} \otimes \hat{\rho}_{\beta}
$$


where $\hat{\rho}_{\beta_{0}}$ and $\hat{\rho}_{\beta}$ are the initial thermal equilibrium density operators. We want to investigate how the initial thermal equilibrium state for the oscillator evolves in time when it is coupled to the environment. More explicitly, we want to study the equilibration process and in particular we would like to know how is the final equilibrium distribution reached and how long it takes to reach that state.

In order to solve for the density operator in an exact way we proceed in an identical way as in last section. It is clear that analogous expressions to those obtained in the last section will also be obtained here, with similar arguments from the $\mathrm{LvN}$ approach being carried out to this problem here. For example, the thermal expectation value of the number operator can be shown that can be put in the form, in terms of Bogoliubov coefficients, as

$$
\bar{n}_{\mu}(t)=\sum_{\nu=0}^{N}\left[\left(\left|\alpha_{\mu \nu}(t)\right|^{2}+\left|\beta_{\nu \mu}(t)\right|^{2}\right) \bar{n}_{\nu}+\left|\beta_{\nu \mu}(t)\right|^{2}\right],
$$

where $\mu=(0, k), k=1,2 .$. and $\bar{n}_{0}, \bar{n}_{k}$ are the initial thermal equilibrium distributions for the oscillator and the environment, respectively, given by

$$
\bar{n}_{0}=\frac{1}{e^{\beta_{0} \tilde{\omega}}-1}
$$

and

$$
\bar{n}_{k}=\frac{1}{e^{\beta \omega_{k}}-1}
$$

Note that in Eq. (94) we have used $\tilde{\omega}$ instead of $\omega_{0}$. As will become clearer in the derivation below, $\omega_{0}$ will represent a bare frequency, while zero point corrections coming from the coupling to the (infinity) environment oscillators leads to the definition of a physical (renormalized) frequency, that here we 
denote it by $\tilde{\omega}$. The Bogoliubov coefficients are given by equations analogous to Eqs. (54)-(55)

$$
\begin{aligned}
& \alpha_{\mu \nu}(t)=i\left(\dot{B}_{\nu \nu}^{*} B_{\mu \nu}(t)-B_{\nu \nu}^{*} \dot{B}_{\mu \nu}(t)\right), \\
& \beta_{\mu \nu}(t)=i\left(B_{\nu \nu} \dot{B}_{\mu \nu}(t)-\dot{B}_{\nu \nu} B_{\mu \nu}(t)\right)
\end{aligned}
$$

where $B_{\mu \nu}$ are the non zero initial values of $B_{\mu \nu}(t)$,

$$
B_{\mu \nu}=\delta_{\mu \nu} \frac{e^{i \tilde{\omega}_{\mu} t}}{\sqrt{2 \tilde{\omega}_{\mu}}}
$$

where $\tilde{\omega}_{\mu}=\left(\tilde{\omega}, \omega_{k}\right)$. Of course, the Bogoliubov coefficients also follow identical relations as given by Eqs. (56)-(57). The coefficients $B_{\mu \nu}(t)$ also obey equations similar to Eqs. (46)-(47),

$$
\begin{aligned}
& \ddot{B}_{\mu 0}(t)+\omega_{0}^{2} B_{\mu 0}(t)+\sum_{k=1}^{N} \lambda_{k}(t) B_{\mu k}(t)=0, \\
& \ddot{B}_{\mu k}(t)+\omega_{k}^{2} B_{\mu k}(t)+\lambda_{k}(t) B_{\mu 0}(t)=0 .
\end{aligned}
$$

In general Eqs. (99)-(100) cannot be solved analytically for any arbitrary time dependence of $\lambda(t)$. There is a situation however in which these equations can be solved exactly, and this is the case when $\lambda(t)$ is suddenly turned on at some given time, for example at $t=0$. For convenience we can then express $\lambda_{k}(t)$ as

$$
\lambda_{k}(t)= \begin{cases}0, & t \leq 0 \\ \omega_{k} \sqrt{g \Delta \omega}, & t>0\end{cases}
$$

where $g$ is now a constant (coupling constant) parameter introduced for convenience and $\Delta \omega$ is defined below. Also in order to simplify our calculations 
we start with $\omega_{k}$ given by

$$
\omega_{k}=\frac{k \pi}{L}, k=1,2, \ldots
$$

and then we go to the continuum limit at the end by letting $L \rightarrow \infty$. In Eq. (101) $\Delta \omega=\frac{\pi}{L}$ is the interval between two neighboring frequencies. For $t<0$ the solution of the system of Eqs. (99)-(100) is given by Eq. (98). For $t>0$ the solution is found by introducing, as in Sec. III, normal coordinates $Q_{\mu \nu}(t)$ now defined by

$$
B_{\mu \nu}(t)=\sum_{\rho=0}^{N} \eta_{\nu \rho} Q_{\mu \rho}(t)
$$

where $Q_{\mu \rho}(t)$ satisfy

$$
\ddot{Q}_{\mu \rho}(t)+\Omega_{\rho}^{2} Q_{\mu \rho}(t)=0
$$

and $\eta_{\mu \nu}$ is an orthogonal matrix with elements (for details see appendix A) given by

$$
\begin{aligned}
& \eta_{k \mu}=\frac{\omega_{k} \sqrt{g \Delta \omega}}{\Omega_{\mu}^{2}-\omega_{k}^{2}} \eta_{0 \mu} \\
& \eta_{0 \mu}=\left[1+\sum_{k=1}^{N} \frac{g \omega_{k}^{2} \Delta \omega}{\left(\omega_{k}^{2}-\Omega_{\nu}^{2}\right)^{2}}\right]^{-\frac{1}{2}},
\end{aligned}
$$

with normal frequencies $\Omega_{\mu}$ obtained as the solutions of

$$
\omega_{0}^{2}-\Omega_{\mu}^{2}=\sum_{k=1}^{N} \frac{g \omega_{k}^{2} \Delta \omega}{\omega_{k}^{2}-\Omega_{\mu}^{2}} .
$$

Eq. (107) can be rewritten as

$$
\omega_{0}^{2}-g N \Delta \omega-\Omega_{\mu}^{2}=\sum_{k=1}^{N} \frac{g \Omega_{\mu}^{2} \Delta \omega}{\omega_{k}^{2}-\Omega_{\mu}^{2}} .
$$


By taking the limit $N \rightarrow \infty$ in Eq. (108) we see that the above equation make no sense, since in such case the left hand side is infinity while the right hand is not. This is a consequence of the (infinity) zero point energy added as we let $N \rightarrow \infty$. In order to overcome this problem we introduce a renormalized frequency $\tilde{\omega}$ defined as

$$
\tilde{\omega}=\sqrt{\omega_{0}^{2}-g N \Delta \omega}
$$

In order to $\tilde{\omega}$ be finite we see that $\omega_{0}^{2}$ have to be infinity. Then we conclude that $\omega_{0}$ is not the physical frequency of the oscillator, it is a bare (unrenormalized) frequency. The physical frequency is the finite renormalized frequency $\tilde{\omega}$. Also the introduction of the renormalized frequency guarantees that all the solutions $\Omega^{2}$ of Eq. (108) be positive allowing us to avoid possible runaway solutions [18].

Using Eq. (102) and taking $N, L \rightarrow \infty$ in Eqs. (105)-(107) we obtain

$$
\begin{aligned}
\eta_{0 \mu} & =\lim _{\Delta \omega \rightarrow 0} \frac{2 \Omega_{\mu} \sqrt{g \Delta \omega}}{\sqrt{4\left(\Omega_{\mu}^{2}-\tilde{\omega}^{2}\right)^{2}+\pi^{2} g^{2} \Omega_{\mu}^{2}}}, \\
\eta_{k \mu} & =\frac{2 g \omega_{k} \Delta \omega}{\left(\Omega_{\mu}^{2}-\omega_{k}^{2}\right)} \frac{\Omega_{\mu}}{\sqrt{4\left(\Omega_{\mu}^{2}-\tilde{\omega}^{2}\right)^{2}+\pi^{2} g^{2} \Omega_{\mu}^{2}}}
\end{aligned}
$$

and

$$
\cot (L \Omega)=\frac{2 \Omega}{\pi g}+\frac{\Delta \omega}{\pi \Omega}\left(1-\frac{2 \tilde{\omega}^{2}}{g \Delta \omega}\right)
$$

where in Eq. (112) we have dropped the index $\mu$ for simplicity and it is to be understood that $\Omega_{\mu}$ are the roots of Eq. (112). 
Substituting the solutions of Eq. (104) in Eq. (103) leads to the following expression for $B_{\mu \nu}(t)$

$$
B_{\mu \nu}(t)=\sum_{\rho=0}^{N} \eta_{\nu \rho}\left(a_{\mu \rho} e^{i \Omega_{\rho} t}+b_{\mu \rho} e^{-i \Omega_{\rho} t}\right)
$$

From the continuity of $B_{\mu \nu}(t)$ and of its first derivative at $t=0$ we can obtain $a_{\mu \rho}$ and $b_{\mu \rho}$ (see appendix A). Substituting the obtained expressions for these coefficients in Eq. (113) we obtain the result

$$
B_{\mu \nu}(t)=\sum_{\rho=0}^{N} \frac{\eta_{\mu \rho} \eta_{\nu \rho}}{\sqrt{8 \tilde{\omega}_{\mu}}}\left[\left(1+\frac{\tilde{\omega}_{\mu}}{\Omega_{\rho}}\right) e^{i \Omega_{\rho} t}+\left(1-\frac{\tilde{\omega}_{\mu}}{\Omega_{\rho}}\right) e^{-i \Omega_{\rho} t}\right],
$$

from which the Bogoliubov coefficients follow

$$
\alpha_{\mu \nu}(t)=\sqrt{\frac{\tilde{\omega}_{\nu}}{2}} B_{\mu \nu}(t)-\frac{i}{\sqrt{2 \tilde{\omega}_{\nu}}} \dot{B}_{\mu \nu}(t)
$$

and

$$
\beta_{\mu \nu}(t)=\sqrt{\frac{\tilde{\omega}_{\nu}}{2}} B_{\mu \nu}(t)+\frac{i}{\sqrt{2 \tilde{\omega}_{\nu}}} \dot{B}_{\mu \nu}(t) .
$$

Taking $\mu=0$ in Eq. (93), we obtain the time dependent thermal expectation value of the number operator for the oscillator $q_{0}$,

$$
\begin{aligned}
\bar{n}_{0}(t)=\left[\left(\left|\alpha_{00}(t)\right|^{2}\right.\right. & \left.+\left|\beta_{00}(t)\right|^{2}\right) \bar{n}_{0}+\left|\beta_{00}(t)\right|^{2} \\
& +\sum_{k}\left[\left(\left|\alpha_{0 k}(t)\right|^{2}+\left|\beta_{k 0}(t)\right|^{2}\right) \bar{n}_{k}+\left|\beta_{k 0}(t)\right|^{2}\right] .
\end{aligned}
$$

Then in order to obtain $\bar{n}_{0}(t)$ we have first to compute $\alpha_{00}(t), \alpha_{0 k}(t), \beta_{00}(t)$ and $\beta_{k 0}(t)$. These coefficients are obtained in Appendix B. They are given by Eqs. (B.6), (B.7), (B.14) and (B.15). From Eqs. (B.6)-(B.7) we can see that

$$
\alpha_{00}(t \rightarrow \infty)=\beta_{00}(t \rightarrow \infty)=0
$$


On the other hand from Eqs. (B.14)-(B.15) we obtain

$$
\begin{aligned}
& \alpha_{0 k}(t \rightarrow \infty)=\sqrt{\frac{\omega_{k}}{\tilde{\omega}}} \frac{\left(\tilde{\omega}+\omega_{k}\right)\left(2 \omega_{k}^{2}-2 \tilde{\omega}^{2}+i \pi g \omega_{k}\right)}{\left[4\left(\omega_{k}^{2}-\tilde{\omega}^{2}\right)^{2}+\pi^{2} g^{2} \omega_{k}^{2}\right]} \sqrt{g \Delta \omega} e^{i \omega_{k} t}, \\
& \beta_{k 0}(t \rightarrow \infty)=\sqrt{\frac{\omega_{k}}{\tilde{\omega}}} \frac{\left(\tilde{\omega}-\omega_{k}\right)\left(2 \omega_{k}^{2}-2 \tilde{\omega}^{2}+i \pi g \omega_{k}\right)}{\left[4\left(\omega_{k}^{2}-\tilde{\omega}^{2}\right)^{2}+\pi^{2} g^{2} \omega_{k}^{2}\right]} \sqrt{g \Delta \omega} e^{i \omega_{k} t} .
\end{aligned}
$$

Now substituting the above equations in Eq. (117) and going to the continuum limit we obtain for $\bar{n}_{0}(t \rightarrow \infty)$ the following result

$$
\begin{array}{r}
\bar{n}_{0}(t \rightarrow \infty)=\frac{2 g}{\tilde{\omega}} \int_{0}^{\infty} d \omega \frac{\omega\left(\omega^{2}+\tilde{\omega}^{2}\right)}{\left[4\left(\omega^{2}-\tilde{\omega}^{2}\right)^{2}+\pi^{2} g^{2} \omega^{2}\right]} \frac{1}{\left(e^{\beta \omega}-1\right)} \\
+\frac{g}{\tilde{\omega}} \int_{0}^{\infty} d \omega \frac{\omega(\omega-\tilde{\omega})^{2}}{4\left(\omega^{2}-\tilde{\omega}^{2}\right)^{2}+\pi^{2} g^{2} \omega^{2}}
\end{array}
$$

Note that, as a consequence of Eq. (118), the asymptotic result for the number density of the system's oscillator $q_{0}$ does not depend on its initial temperature, only on the (continuum) ensemble initial temperature $T=1 / \beta$. We also note that while the first term on the right-hand-side in Eq. (121) is convergent, the second term diverges logarithmically for $\omega \rightarrow \infty$. It is easy to prove that this divergent term is equal to the asymptotic value of $\left\langle 0\left|\hat{a}_{0}^{\dagger}(t) \hat{a}_{0}(t)\right| 0\right\rangle$, where $|0\rangle$ means the initial ground state of the system, i.e, the state in which all the harmonic oscillators are in their ground states. Then we can interpret the divergent term as the particles of frequency $\tilde{\omega}$ produced by the modification of the initial ground state. Clearly this term does not have a thermal origin. It is a response of the ground state to the interaction. In computing the final equilibrium temperature we have to use only the part of $\bar{n}_{0}(t \rightarrow \infty)$ that have a thermal origin and this corresponds to the first (finite) term in Eq. (121). Alternatively note also that the last term in Eq. (121) can be eliminated by making a normal ordering of the initial annihilation and creation operators. 
The distribution given by Eq. (121) is one of equilibrium, but how is it associated to a distribution of thermal equilibrium as obtained from a thermal expectation value for the system's number operator ? For this, let us start by computing the thermal expectation value of the number operator corresponding to the particle oscillator by assuming that it is in thermal equilibrium, at some temperature $1 / \beta^{\prime}$, with the thermal bath. We then have that at thermal equilibrium the density operator for the total system is

$$
\hat{\rho}_{\beta^{\prime}}=\frac{e^{-\beta^{\prime} \hat{H}}}{\operatorname{Tr}\left(e^{-\beta^{\prime} \hat{H}}\right)}
$$

where $\hat{H}$ is given by Eq. (91) evaluated at $t \rightarrow \infty$, that we here express it directly in its diagonal form:

$$
\hat{H}=\sum_{\mu=0}^{N}\left[\hat{A}_{\mu}^{\dagger} \hat{A}_{\mu}+\frac{1}{2}\right] \Omega_{\mu}
$$

where $\hat{A}_{\mu}^{\dagger}$ and $\hat{A}_{\mu}$ are the creation and annihilation operators associated with the normal coordinates that diagonalizes the original Hamiltonian and whose diagonalization transformation between the coordinates $q_{\mu}$ and normal coordinates $Q_{\mu}$ is given by the same matrix transformation $\eta_{\mu \nu}$, of Eqs. (110) and (111)

$$
q_{\mu}=\sum_{\nu=0}^{N} \eta_{\mu \nu} Q_{\nu}
$$

Using Eq. (124) it is easy to show that the annihilation and creation operators, $\hat{a}_{\mu}$ and $\hat{a}_{\mu}^{\dagger}$, associates to the coordinates $q_{\mu}$, in terms of $\hat{A}_{\mu}^{\dagger}$ and $\hat{A}_{\mu}$ are given by

$$
\hat{a}_{\mu}=\sum_{\nu=0}^{N} \frac{\eta_{\mu \nu}}{2}\left[\sqrt{\frac{\tilde{\omega}_{\mu}}{\Omega_{\nu}}}\left(\hat{A}_{\nu}+\hat{A}_{\nu}^{\dagger}\right)+\sqrt{\frac{\Omega_{\nu}}{\tilde{\omega}_{\mu}}}\left(\hat{A}_{\nu}-\hat{A}_{\nu}^{\dagger}\right)\right]
$$


and

$$
\hat{a}_{\mu}^{\dagger}=\sum_{\nu=0}^{N} \frac{\eta_{\mu \nu}}{2}\left[\sqrt{\frac{\tilde{\omega}_{\mu}}{\Omega_{\nu}}}\left(\hat{A}_{\nu}+\hat{A}_{\nu}^{\dagger}\right)+\sqrt{\frac{\Omega_{\nu}}{\tilde{\omega}_{\mu}}}\left(\hat{A}_{\nu}^{\dagger}-\hat{A}_{\nu}\right)\right],
$$

where $\bar{\omega}_{\mu}=\left(\tilde{\omega}, \omega_{k}\right)$. We can now compute the interacting system's equilibrium number distribution, that we denote as $\bar{n}_{0}^{\prime}$, which is given by

$$
\bar{n}_{0}^{\prime}=\frac{\operatorname{Tr}\left(\hat{a}_{0}^{\dagger} \hat{a}_{0} e^{-\beta^{\prime} \hat{H}}\right)}{\operatorname{Tr}\left(e^{-\beta^{\prime} \hat{H}}\right)} .
$$

Substituting Eq. (123) and Eqs. (125)-(126) with $\mu=0$ in Eq. (127) we get

$$
\bar{n}_{0}^{\prime}=\frac{1}{2} \sum_{\mu=0}^{N} \frac{\eta_{0 \mu}^{2}}{\tilde{\omega} \Omega_{\mu}} \frac{\left(\Omega_{\mu}^{2}+\tilde{\omega}^{2}\right)}{\left(e^{\beta^{\prime} \Omega_{\mu}}-1\right)}+\frac{1}{4} \sum_{\mu=0}^{N} \frac{\eta_{0 \mu}^{2}}{\tilde{\omega} \Omega_{\mu}}\left(\Omega_{\mu}-\tilde{\omega}\right)^{2} .
$$

Finally, by taking $N \rightarrow \infty, L \rightarrow \infty$ and using Eq. (110) in Eq. (128) we obtain, in the limit of the continuum, that

$$
\begin{array}{r}
\bar{n}_{0}^{\prime}=\frac{2 g}{\tilde{\omega}} \int_{0}^{\infty} d \Omega \frac{\Omega\left(\Omega^{2}+\tilde{\omega}^{2}\right)}{\left[4\left(\Omega^{2}-\tilde{\omega}^{2}\right)^{2}+\pi^{2} g^{2} \Omega^{2}\right]} \frac{1}{\left(e^{\beta^{\prime} \Omega}-1\right)} \\
+\frac{g}{\tilde{\omega}} \int_{0}^{\infty} d \Omega \frac{\Omega(\Omega-\tilde{\omega})^{2}}{4\left(\Omega^{2}-\tilde{\omega}^{2}\right)^{2}+\pi^{2} g^{2} \Omega^{2}}
\end{array}
$$

Note that the above equation is identical to Eq. (121) if we identify $\beta^{\prime}=$ $\beta$. Then, we have showed that the particle oscillator reachs a final thermal equilibrium state at a temperature $T_{f}$ that is exactly equal to that of the thermal bath, T. By introducing dimensionless parameters, $\bar{g}=\frac{g}{\tilde{\omega}}$ and $\bar{\beta}=\tilde{\omega} \beta$, we can write for the finite part of Eq. (121), that we denote as $\bar{n}_{\text {eq }}$, the following expression

$$
\bar{n}_{\mathrm{eq}}=2 \tilde{g} \int_{0}^{\infty} d x \frac{x\left(x^{2}+1\right)}{\left[4\left(x^{2}-1\right)^{2}+\pi^{2} \tilde{g}^{2} x^{2}\right]\left(e^{\tilde{\beta} x}-1\right)} .
$$


In Fig. 3 we show the numerical results for $\bar{n}_{\text {eq }}$, normalized by a Bose-Einstein distribution at temperature $T$ and energy $\tilde{\omega}$, as a function of scaled temperature and coupling constants, $T / \tilde{\omega}$ and $g / \tilde{\omega}$, respectively. The results shown in Fig. 3 show that Eq. (130) quickly approaches to the distribution $\tilde{n}_{0}=$ $1 /[\exp (\beta \tilde{\omega})-1]$ at high temperatures $T / \tilde{\omega}>1$.

\section{Nonlinearities at Gaussian level}

In this section we shall consider the effect of the nonlinearities at the Gaussian approximation. The model that we consider is described by the following Hamiltonian with quartic potential,

$$
\hat{H}=\frac{\hat{p}^{2}}{2}+\frac{\omega^{2}(t)}{2} \hat{q}^{2}+\frac{\lambda(t)}{4 !} \hat{q}^{4}
$$

As in Sec. II we introduce time dependent annihilation and creation operators,

$$
\hat{a}(t)=A(t) \hat{q}+B(t) \hat{p}
$$

and

$$
\hat{a}^{\dagger}(t)=A(t)^{*}(t) \hat{q}+B^{*}(t) \hat{p}
$$

from which using Eq. (7) we can solve for $\hat{q}$ and $\hat{p}$ in terms of $\hat{a}(t)$ and $\hat{a}^{\dagger}(t)$, obtaining

$$
\hat{q}=i\left[B^{*}(t) \hat{a}(t)-B(t) \hat{a}^{\dagger}(t)\right]
$$


and

$$
\hat{p}=-i\left[A^{*}(t) \hat{a}(t)-A(t) \hat{a}^{\dagger}(t)\right] .
$$

Replacing Eqs. (134) and (135) in Eq. (131), normal ordering the time dependent annihilation and creation operators and truncating the Hamiltonian up to quadratic terms in these operators we obtain the result

$$
\begin{aligned}
\hat{H}_{G}=\left(|A(t)|^{2}+\omega^{2}(t)|B(t)|^{2}+\frac{\lambda(t)}{2}|B(t)|^{4}\right) \hat{a}^{\dagger}(t) \hat{a}(t) \\
-\left(A^{2}(t)+\omega^{2}(t) B^{2}(t)+\frac{\lambda(t)}{2} B^{\star}(t) B^{3}(t)\right) \frac{\left[\hat{a}^{\dagger}(t)\right]^{2}}{2} \\
-\left(\left[A^{\star}(t)\right]^{2}+\omega^{2}(t)\left[B^{\star}(t)\right]^{2}+\frac{\lambda(t)}{2} B(t)\left[B^{\star}(t)\right]^{3}\right) \frac{[\hat{a}(t)]^{2}}{2} .
\end{aligned}
$$

Replacing $\hat{a}(t)$ in the LvN equation and using for $\hat{H}$ its Gaussian approximation, as given by Eq. (136), we obtain the following equations for $A(t)$ and $B(t)$,

$$
A(t)=-\dot{B}(t)
$$

and

$$
\ddot{B}(t)+\omega^{2}(t) B(t)+\frac{\lambda(t)}{2}|B(t)|^{2} B(t)=0 .
$$

Note that now $B(t)$ is described by a nonlinear equation, that is, this shows how at the Gaussian level approximation it is possible to include the nonlinearities of the model. As in Sec. II, the time dependent thermal average for the number occupation operator is given by Eq. (25), where the Bogoliubov coefficients are given by the same relations of the Sec. II, the only difference is that now $B(t)$ is described by the nonlinear equation (138). 
In general Eq. (138) cannot be solved exactly. But we can extract some qualitative conclusions in the case in which the frequency is time independent and when $\lambda(t)$ evolves just like as in Eq. (63). In this case we have that

$$
B(t \leq 0)=\frac{\mathrm{e}^{\mathrm{i} \omega \mathrm{t}}}{\sqrt{2 \omega}}
$$

and for $t \geq 0, B(t)$ is described by

$$
\ddot{B}(t)+\omega^{2} B(t)+\frac{\lambda}{2}|B(t)|^{2} B(t)=0 .
$$

We have to solve the above nonlinear equation with the initial condition (at $t=0)$ given from Eq. (139),

$$
B=\frac{1}{\sqrt{2 \omega}}, \quad \dot{B}=i \sqrt{\frac{\omega}{2}} .
$$

To solve Eq. (140), it is useful to express $B(t)$ in the polar form,

$$
B(t)=\psi(t) e^{i \phi(t)},
$$

where $\psi(t)$ and $\phi(t)$ are real functions. Substituting Eq. (142) in Eq. (141) and identifying real and imaginary parts of the resultant equation we obtain the coupled set of differential equations

$$
\begin{aligned}
& \ddot{\psi}(t)+\left(\omega^{2}-[\dot{\phi}(t)]^{2}\right) \psi(t)+\frac{\lambda}{2} \psi^{3}(t)=0, \\
& 2 \dot{\psi}(t) \dot{\phi}(t)+\psi(t) \ddot{\phi}(t)=0 .
\end{aligned}
$$

From Eq. (144) we immediatly obtain that

$$
\dot{\phi}(t)=c \psi^{-2}(t),
$$


where $c$ is a constant that can be fixed by using the initial conditions. Comparing Eqs. (139) and Eqs. (142), we conclude that at $t=0, \psi=1 / \sqrt{2 \omega}, \dot{\psi}=0$, $\phi=0$ and $\dot{\phi}=\omega$. Using these values in Eq. (145) we obtain that $c=1 / 2$. Then integrating Eq. (145) with the initial condition $\phi=0$ we obtain,

$$
\phi(t)=\frac{1}{2} \int_{0}^{t} d t^{\prime} \psi^{-2}\left(t^{\prime}\right) .
$$

To solve for $\psi(t)$ we replace Eq. (145) (with $c=1 / 2$ ) in Eq. (143) to obtain the result

$$
\ddot{\psi}(t)+\omega^{2} \psi(t)-\frac{1}{4} \psi^{-3}(t)+\frac{\lambda}{2} \psi^{3}(t)=0
$$

Note that the above equation is singular for $\psi=0$, but as we shall show below, this singularity is never reached by $\psi(t)$ in the course of its time evolution. Using Eqs. (21) and (22) in Eq. (25) and replacing $B(t)$ as given by Eq. (142), we obtain for the time dependent thermal expectation value of the number operator, $\bar{n}(t)$, the following expression

$$
\begin{aligned}
\bar{n}(t)=\left[\dot{\psi}^{2}(t)+\omega^{2} \psi^{2}(t)\right. & \left.+\frac{1}{4} \psi^{-2}(t)\right] \frac{\bar{n}}{\omega} \\
& +\frac{1}{2}\left[\dot{\psi}^{2}(t)+\omega^{2} \psi^{2}(t)+\frac{1}{4} \psi^{-2}(t)\right]-\frac{1}{2}
\end{aligned}
$$

Then, in order to know the time evolution of $\bar{n}(t)$ we only need to know the time evolution of $\psi(t)$. From Eq. (147) and using the initial conditions for $\psi(t)$ and $\dot{\psi}(t)$ we obtain

$$
\frac{1}{2} \dot{\psi}^{2}(t)+\frac{\omega^{2}}{2} \psi^{2}(t)+\frac{1}{8} \psi^{-2}(t)+\frac{\lambda}{8} \psi^{4}(t)=\frac{\omega}{2}+\frac{\lambda}{32 \omega^{2}} .
$$


from which we get

$$
t= \pm \int_{1 / 2 \omega}^{\psi^{2}(t)} \frac{d x}{\sqrt{-\frac{1}{4}+\left(\omega+\frac{\lambda}{16 \omega^{2}}\right) x-\omega^{2} x^{2}-\frac{\lambda}{4} x^{3}}}
$$

The above integral cannot be solved analytically, and then we are not able to solve for $\psi(t)$. But we can obtain some qualitative conclusions about the behavior in time of $\psi(t)$ that will help us to understand the effect of nonlinearities on the thermal equilibration of the system we are studying. For this end we rewrite Eq. (148) by using Eq. (149), obtaining

$$
\bar{n}(t)=\left(1+\frac{\lambda}{16 \omega^{3}}-\frac{\lambda}{4 \omega} \psi^{4}(t)\right) \bar{n}+-\frac{\lambda}{8 \omega} \psi^{4}(t)+\frac{\lambda}{32 \omega^{3}} .
$$

Then, if we know the qualitative behavior of $\psi(t)$ we would equally well know the qualitative behavior of $\bar{n}(t)$. If in Eq. (149) we think in $\psi(t)$ as being the analogous of the coordinate of a particle, then Eq. (149) is nothing but the energy conservation law of a particle of unity mass that moves in a conservative potential $U(\psi)$ given by

$$
U(\psi)=\frac{\omega^{2}}{2} \psi^{2}+\frac{1}{8} \psi^{-2}+\frac{\lambda}{8} \psi^{4}
$$

It is clear from the potential $U(\psi)$ that for any finite energy $\psi(t)$ evolve in time in an oscillatory way, never reaching a fixed value, and therefore the system never reaches a final thermal equilibrium state. Note also that in order to $\psi(t)$ to reach the singularity $\psi=0$, the energy, in the particle mechanical analogy, need to be infinity. Since the energy, as given by the right hand side of Eq. (149), is finite, we also conclude that such singularity can never be reached. 


\section{Conclusions}

In this work we have studied several simple nonequilibrium models represented by quantum oscillators in interaction that can be exactly solved. In special we have studied examples of smooth and sudden quenches that were explicitly solved in the context of the LvN approach. As emphasized in Refs. [11,12], the LvN approach has a clear advantage over other techniques to study out-ofthermal equilibrium problems since standard methods of quantum mechanics are used. We have explicitly seen this here by working directly in terms of annihilation and creation operators and their Bogoliubov transformation relations, allowing to determine the evolution of number densities among other quantities, like the final equilibration temperatures and the general system time evolution.

Previous use of the method, like to the study of second order phase transitions [12], for the decoherence problem [19] and for the studies performed here, show that the LvN approach can easily be adapted to study more complex problems that are difficult to deal with in the context of nonequilibrium dynamics of fields where usually the expressions for nonequilibrium propagators and correlation functions must be worked out. In this case the $\mathrm{LvN}$ approach may provide an avenue to more rigorously take into account in the dynamics of systems the interactions with the environment degrees of freedom. An example of this has been worked out in details in Sec. IV, for the model of the system quantum oscillator in interaction with an infinity set of other quantum oscillators.

The extension of the method for applications to the current important prob- 
lems of decoherence and description of dissipative dynamics of quantum systems, for the more realistic cases where nonlinear interactions are present, is currently in progress and we expect to report on them in the near future.

\section{Acknowledgments}

R.O.R. was partially supported by Conselho Nacional de Desenvolvimento Científico e Tecnológico - CNPq (Brazil). G.F.H was supported by a grant from CNPq.

\section{A}

In this appendix we solve the system of equations given by

$$
\begin{aligned}
& \ddot{B}_{\mu 0}(t)+\omega_{0}^{2} B_{\mu 0}(t)+\sum_{k=1}^{N} \lambda_{k} B_{\mu k}(t)=0, \\
& \ddot{B}_{\mu k}(t)+\omega_{k}^{2} B_{\mu k}(t)+\lambda_{k} B_{\mu 0}(t)=0,
\end{aligned}
$$

with initial conditions at $t=0$ given by

$$
B_{\mu \nu}=\frac{\delta_{\mu \nu}}{2 \omega_{\mu}}, \quad \dot{B}_{\mu \nu}=i \delta_{\mu \nu} \sqrt{\frac{\omega_{\mu}}{2}}
$$

When $N=1, k=1$ and then we can write $\lambda_{k}=\lambda$ the equations above become identical to Eqs. (46)-(47) with time independent coupling constant (and changing the indexes 0,1 to 1,2 ). On the other hand, when $\lambda_{k}=\omega_{k} \sqrt{g \Delta \omega}$ we recover Eqs. (99)-(100) with time independent coupling constant as given by Eq. (101). Also in this last case, in the initial conditions given by Eq. (A.3) we have to replace the bare frequencies $\omega_{\mu}$ by the renormalized ones 
$\tilde{\omega}_{\mu}=\left(\tilde{\omega}, \omega_{k}\right)$ [see Eq. (98)]. In order to solve Eqs. (A.1)-(A.2) we introduce normal coordinates $Q_{\mu \nu}(t)$ through

$$
B_{\mu \nu}(t)=\sum_{\rho=0}^{N} \eta_{\nu \rho} Q_{\mu \rho}(t),
$$

where only the second index of $B_{\mu \nu}(t)$ appear in the matrix transformation $\{\eta\}$ since the first index are uncoupled in Eqs. (A.1)-(A.2). Substituting Eq. (A.4) in Eqs. (A.1)-(A.2) we obtain

$$
\begin{aligned}
& \sum_{\rho=0}^{N}\left[\eta_{0 \rho} \ddot{Q}_{\mu \rho}(t)+\omega_{0}^{2} \eta_{0 \rho} Q_{\mu \rho}(t)+\sum_{k=1}^{N} \lambda_{k} \eta_{k \rho} Q_{\mu \rho}(t)\right]=0, \\
& \sum_{\rho=0}^{N}\left[\eta_{k \rho} \ddot{Q}_{\mu \rho}(t)+\omega_{k}^{2} \eta_{k \rho} Q_{\mu \rho}(t)+\lambda_{k} \eta_{0 \rho} Q_{\mu \rho}(t)\right]=0 .
\end{aligned}
$$

Multiplying Eq. (A.5) by $\eta_{0 \sigma}$ and Eq. (A.6) by $\eta_{k \sigma}$ and adding the resulting equations we obtain

$$
\begin{aligned}
\sum_{\rho=0}^{N}\left\{\left[\sum_{\nu=0}^{N} \omega_{\nu}^{2} \eta_{\nu \rho} \eta_{\nu \sigma}+\sum_{k=1}^{N} \lambda_{k}\left(\eta_{k \rho} \eta_{0 \sigma}\right.\right.\right. & \left.\left.+\eta_{0 \rho} \eta_{k \sigma}\right)\right] Q_{\mu \rho}(t) \\
& \left.+\sum_{\nu=0}^{N} \eta_{\nu \rho} \eta_{\nu \sigma} \ddot{Q}_{\mu \rho}(t)\right\}=0,
\end{aligned}
$$

and imposing that,

$$
\begin{aligned}
& \sum_{\nu=0}^{N} \eta_{\nu \rho} \eta_{\nu \sigma}=\delta_{\rho \sigma} \\
& \sum_{\nu=0}^{N} \omega_{\nu}^{2} \eta_{\nu \rho} \eta_{\nu \sigma}+\sum_{k=1}^{N} \lambda_{k}\left(\eta_{k \rho} \eta_{0 \sigma}+\eta_{0 \rho} \eta_{k \sigma}\right)=\delta_{\rho \sigma} \Omega_{\sigma}^{2},
\end{aligned}
$$

we obtain

$$
\ddot{Q}_{\mu \sigma}(t)+\Omega_{\sigma}^{2} Q_{\mu \sigma}(t)=0 .
$$


Eq. (A.9) can also be written as

$$
\sum_{\nu=0}^{N}\left[\omega_{\nu}^{2} \eta_{\nu \rho}+\sum_{k=1}^{N} \lambda_{k}\left(\eta_{k \rho} \delta_{0 \nu}+\eta_{0 \rho} \delta_{k \nu}\right)\right] \eta_{\nu \sigma}=\delta_{\rho \sigma} \Omega_{\sigma}^{2}
$$

From Eqs. (A.8) and (A.11) we obtain that

$$
\omega_{\nu}^{2} \eta_{\nu \rho}+\sum_{k=1}^{N} \lambda_{k}\left(\eta_{k \rho} \delta_{0 \nu}+\eta_{0 \rho} \delta_{k \nu}\right)=\eta_{\nu \rho} \Omega_{\rho}^{2}
$$

Taking $\nu=0$ in the above equation we obtain that

$$
\omega_{0}^{2} \eta_{0 \rho}+\sum_{k=1}^{N} \lambda_{k} \eta_{k \rho}=\eta_{0 \rho} \Omega_{\rho}^{2}
$$

while for $\nu \neq 0$ we obtain for $\eta_{k \rho}$ the result

$$
\eta_{k \rho}=\frac{\lambda_{k}}{\Omega_{\rho}^{2}-\omega_{k}^{2}} \eta_{0 \rho}
$$

Taking $\rho=\sigma$ in Eq. (A.8) and using Eq. (A.14) we can solve for $\eta_{0 \rho}$ obtaining the expression

$$
\eta_{0 \rho}=\left[1+\sum_{k=1}^{N} \frac{\lambda_{k}^{2}}{\left(\omega_{k}^{2}-\Omega_{\rho}\right)^{2}}\right]^{-\frac{1}{2}} .
$$

Using Eq. (A.14) in Eq. (A.13) we also get that

$$
\omega_{0}^{2}-\Omega_{\rho}^{2}=\sum_{k=1}^{N} \frac{\lambda_{k}^{2}}{\omega_{k}^{2}-\Omega_{\rho}^{2}}
$$

Now, from Eq. (A.10) we can write for $B_{\mu \nu}(t)$ the following expression

$$
B_{\mu \nu}(t)=\sum_{\rho=0}^{N} \eta_{\nu \rho}\left(a_{\mu \rho} e^{i \Omega_{\rho} t}+b_{\mu \rho} e^{-i \Omega_{\rho} t}\right),
$$

and using the initial conditions given by Eq. (A.3) we get for $a_{\mu \rho}$ and $b_{\mu \rho}$, respectively, the equations 


$$
\begin{aligned}
& a_{\mu \nu}=\frac{\eta_{\mu \nu}}{\sqrt{8 \omega_{\mu}}}\left(1+\frac{\omega_{\mu}}{\Omega_{\nu}}\right) \\
& b_{\mu \nu}=\frac{\eta_{\mu \nu}}{\sqrt{8 \omega_{\mu}}}\left(1-\frac{\omega_{\mu}}{\Omega_{\nu}}\right) .
\end{aligned}
$$

Substituting Eq. (A.18) in Eq. (A.17) we finally obtain the result

$$
B_{\mu \nu}(t)=\sum_{\rho=0}^{N} \frac{\eta_{\mu \rho} \eta_{\nu \rho}}{\sqrt{8 \omega_{\mu}}}\left[\left(1+\frac{\omega_{\mu}}{\Omega_{\rho}}\right) \mathrm{e}^{\mathrm{i} \Omega_{\rho} \mathrm{t}}+\left(1-\frac{\omega_{\mu}}{\Omega_{\rho}}\right) \mathrm{e}^{-\mathrm{i} \Omega_{\rho} \mathrm{t}}\right] .
$$

B

In this section we deduce the Bogoliubov coefficients $\alpha_{00}(t), \beta_{00}(t), \alpha_{0 k}(t)$, and $\beta_{k 0}(t)$. Using Eq. (114) in Eqs. (115)-(116) we obtain

$$
\begin{array}{r}
\alpha_{\mu \nu}(t)=\sum_{\rho=0}^{N}\left\{\sqrt{\frac{\tilde{\omega}_{\nu}}{\tilde{\omega}_{\mu}}} \frac{\eta_{\mu \rho} \eta_{\nu \rho}}{4 \Omega_{\rho}}\left[\left(\Omega_{\rho}+\tilde{\omega}_{\mu}\right) \mathrm{e}^{\mathrm{i} \Omega_{\rho} \mathrm{t}}+\left(\Omega_{\rho}-\tilde{\omega}_{\mu}\right) \mathrm{e}^{-\mathrm{i} \Omega_{\rho} \mathrm{t}}\right]\right. \\
\left.+\frac{\eta_{\mu \rho} \eta_{\nu \rho}}{4 \sqrt{\tilde{\omega}_{\mu} \tilde{\omega}_{\nu}}}\left[\left(\tilde{\omega}_{\mu}+\Omega_{\rho}\right) \mathrm{e}^{\mathrm{i} \Omega_{\rho} \mathrm{t}}+\left(\tilde{\omega}_{\mu}-\Omega_{\rho}\right) \mathrm{e}^{-\mathrm{i} \Omega_{\rho} \mathrm{t}}\right]\right\}, \\
\beta_{\mu \nu}(t)=\sum_{\rho=0}^{N}\left\{\sqrt{\frac{\tilde{\omega}_{\nu}}{\tilde{\omega}_{\mu}}} \frac{\eta_{\mu \rho} \eta_{\nu \rho}}{4 \Omega_{\rho}}\left[\left(\Omega_{\rho}+\tilde{\omega}_{\mu}\right) \mathrm{e}^{\mathrm{i} \Omega_{\rho} \mathrm{t}}+\left(\Omega_{\rho}-\tilde{\omega}_{\mu}\right) \mathrm{e}^{-\mathrm{i} \Omega_{\rho} \mathrm{t}}\right]\right. \\
\left.-\frac{\eta_{\mu \rho} \eta_{\nu \rho}}{4 \sqrt{\tilde{\omega}_{\mu} \tilde{\omega}_{\nu}}}\left[\left(\tilde{\omega}_{\mu}+\Omega_{\rho}\right) \mathrm{e}^{\mathrm{i} \Omega_{\rho} \mathrm{t}}+\left(\tilde{\omega}_{\mu}-\Omega_{\rho}\right) \mathrm{e}^{-\mathrm{i} \Omega_{\rho} \mathrm{t}}\right]\right\} .
\end{array}
$$

Taking $\mu, \nu=0$ in the above equations we obtain

$$
\begin{aligned}
& \alpha_{00}=\sum_{\rho=0}^{N} \frac{\left(\eta_{0 \rho}\right)^{2}}{4}\left[\left(2+\frac{\tilde{\omega}}{\Omega_{\rho}}+\frac{\Omega_{\rho}}{\tilde{\omega}}\right) e^{i \Omega_{\rho} t}+\left(2-\frac{\tilde{\omega}}{\Omega_{\rho}}-\frac{\Omega_{\rho}}{\tilde{\omega}}\right) e^{-i \Omega_{\rho} t}\right], \\
& \beta_{00}=\sum_{\rho=0}^{N} \frac{\left(\eta_{0 \rho}\right)^{2}}{4}\left[\left(\frac{\tilde{\omega}}{\Omega_{\rho}}-\frac{\Omega_{\rho}}{\tilde{\omega}}\right) e^{i \Omega_{\rho} t}+\left(\frac{\Omega_{\rho}}{\tilde{\omega}}-\frac{\tilde{\omega}}{\Omega_{\rho}}\right) e^{-i \Omega_{\rho} t}\right] .
\end{aligned}
$$

Substituting Eq. (110) in the above equations and going to the continuum limit we obtain, e.g. for $\alpha_{00}(t)$, the expression 


$$
\begin{aligned}
\alpha_{00}(t) & =\frac{g}{\tilde{\omega}} \int_{0}^{\infty} d \Omega \frac{\Omega\left[(\tilde{\omega}+\Omega)^{2} e^{i \Omega t}-(\tilde{\omega}-\Omega)^{2} e^{-i \Omega t}\right]}{4\left(\Omega^{2}-\tilde{\omega}^{2}\right)^{2}+\pi^{2} g^{2} \Omega^{2}} \\
& =\frac{g}{2 \tilde{\omega}} \int_{-\infty}^{\infty} d \Omega \frac{\Omega\left[(\tilde{\omega}+\Omega)^{2} e^{i \Omega t}-(\tilde{\omega}-\Omega)^{2} e^{-i \Omega t}\right]}{4\left(\Omega^{2}-\tilde{\omega}^{2}\right)^{2}+\pi^{2} g^{2} \Omega^{2}} \\
& =\frac{g}{\tilde{\omega}} \int_{-\infty}^{\infty} d \Omega \frac{\Omega(\tilde{\omega}+\Omega)^{2}}{4\left(\Omega^{2}-\tilde{\omega}^{2}\right)^{2}+\pi^{2} g^{2} \Omega^{2}} e^{i \Omega t} .
\end{aligned}
$$

The integral in Eq. (B.5) can be easily evaluated using the Cauchy theorem and choosing as contour of integration in the upper half plane. The poles located in the upper half plane are $\pm \tilde{\tilde{\omega}}+\frac{i \pi g}{4}$, where $\tilde{\tilde{\omega}}=\sqrt{\tilde{\omega}^{2}-\frac{\pi^{2} g^{2}}{16}}$. Assuming $\tilde{\omega}>\frac{\pi g}{4}$ the result is

$$
\alpha_{00}(t)=\frac{e^{-\pi g t / 4}}{64 \tilde{\omega} \tilde{\tilde{\omega}}}\left[(4 \tilde{\omega}+4 \tilde{\tilde{\omega}}+i \pi g)^{2} e^{i \tilde{\tilde{\omega}} t}-(4 \tilde{\omega}-4 \tilde{\tilde{\omega}}+i \pi g)^{2} e^{-i \tilde{\tilde{\omega}} t}\right] .
$$

In the same way we obtain from Eq. (B.4) the expression for $\beta_{00}(t)$ (assuming again $\left.\tilde{\omega}>\frac{\pi g}{4}\right)$,

$$
\beta_{00}(t)=\frac{\pi g e^{-\pi g t / 4}}{32 \tilde{\omega} \tilde{\tilde{\omega}}}\left[(\pi g-4 i \tilde{\tilde{\omega}}) e^{i \tilde{\tilde{\omega}} t}-(\pi g+4 i \tilde{\tilde{\omega}}) e^{-i \tilde{\tilde{\omega}} t}\right] .
$$

Setting $\mu=0, \nu=k$ in Eq. (B.1) we obtain,

$$
\begin{aligned}
& \alpha_{0 k}(t)=\frac{\omega_{k} g \sqrt{g \Delta \omega}}{\sqrt{\tilde{\omega} \omega_{k}}} \sum_{\rho} \frac{\Delta \omega \Omega}{\Omega_{\rho}^{2}-\omega_{k}^{2}}\left[\frac{\left(\omega_{k}+\Omega_{\rho}\right)\left(\Omega_{\rho}+\tilde{\omega}\right) e^{i \Omega_{\rho} t}}{4\left(\Omega^{2}-\tilde{\omega}^{2}\right)^{2}+\pi^{2} g^{2} \Omega^{2}}\right. \\
& \left.+\frac{\left(\omega_{k}-\Omega_{\rho}\right)\left(\Omega_{\rho}-\tilde{\omega}\right) e^{-i \Omega_{\rho} t}}{4\left(\Omega^{2}-\tilde{\omega}^{2}\right)^{2}+\pi^{2} g^{2} \Omega^{2}}\right] .
\end{aligned}
$$

In going to the continuum limit we have to take care since there will appear a singularity when $\Omega=\omega_{k}$. In reality this singularity is fictitious and it can be eliminated from Eq. (B.8) using Eq. (108). In terms of the renormalized frequency $\tilde{\omega}$ Eq. (108) can be written as (setting $\mu=k$ )

$$
\tilde{\omega}^{2}-\Omega_{k}^{2}=\sum_{q \neq k} \frac{g \Omega_{k}^{2} \Delta \omega}{\omega_{q}^{2}-\Omega_{k}^{2}}+\frac{g \Omega_{k}^{2} \Delta \omega}{\omega_{k}^{2}-\Omega_{k}^{2}}
$$




$$
=g \int_{0}^{\infty} d^{\prime} \omega \frac{\Omega_{k}^{2}}{\omega^{2}-\Omega_{k}^{2}}+\frac{g \Omega_{k}^{2} \Delta \omega}{\omega_{k}^{2}-\Omega_{k}^{2}},
$$

where we have isolated the pole at $\omega_{k}=\Omega_{k}$ and the prime in the integral means that the point $\omega=\Omega_{k}$ has been excluded from the integration. It is easy to show that such integration is zero. For this end we write such integration as

$$
I=\frac{g}{2} \int_{-\infty}^{\infty} d^{\prime} \omega \frac{\Omega_{k}^{2}}{\omega^{2}-\Omega_{k}^{2}},
$$

where now it is understood that we omit the points $\pm \Omega_{k}=\omega_{k}$ in doing the integration over the frequency. Going to the complex plane and using Cauchy theorem we obtain,

$$
I=\frac{g \Omega_{k}^{2}}{2}\left(\frac{i \pi}{2 \Omega_{k}}+\frac{i \pi}{-2 \Omega_{k}}\right)=0 .
$$

Then, from Eq. (B.9) we obtain

$$
\tilde{\omega}^{2}-\Omega_{k}^{2}=\frac{g \Omega_{k}^{2} \Delta \omega}{\omega_{k}^{2}-\Omega_{k}^{2}},
$$

where it is understood that $\Omega_{k}=\omega_{k}$ and since we are considering the continuum limit, in which case $\Delta \omega \rightarrow 0$, then the right-hand-side of Eq. (B.12) is well defined. Now we can use Eq. (B.12) in Eq. (B.8) to eliminate the singularity after isolating it. Isolating the singularity in Eq. (B.8) and going to the continuum limit we obtain

$$
\begin{aligned}
\alpha_{0 k}(t)=\frac{\omega_{k} g \sqrt{g \Delta \omega}}{\sqrt{\tilde{\omega} \omega_{k}}} \int_{0}^{\infty} d^{\prime} \Omega \frac{\Omega}{\Omega_{\rho}^{2}-\omega_{k}^{2}}\left[\frac{\left(\omega_{k}+\Omega\right)(\Omega+\tilde{\omega}) e^{i \Omega t}}{4\left(\Omega^{2}-\tilde{\omega}^{2}\right)^{2}+\pi^{2} g^{2} \Omega^{2}}\right. \\
\left.+\frac{\left(\omega_{k}-\Omega\right)(\Omega-\tilde{\omega}) e^{-i \Omega t}}{4\left(\Omega^{2}-\tilde{\omega}^{2}\right)^{2}+\pi^{2} g^{2} \Omega^{2}}\right]+\frac{\omega_{k} g \sqrt{g \Delta \omega}}{\sqrt{\tilde{\omega} \omega_{k}}} \frac{\Delta \omega \Omega_{k}}{\Omega_{k}^{2}-\omega_{k}^{2}} \\
\quad \times\left[\frac{\left(\omega_{k}+\Omega_{k}\right)\left(\Omega_{k}+\tilde{\omega}\right) e^{i \Omega_{k} t}}{4\left(\Omega_{k}^{2}-\tilde{\omega}^{2}\right)^{2}+\pi^{2} g^{2} \Omega_{k}^{2}}+\frac{\left(\omega_{k}-\Omega_{k}\right)\left(\Omega_{k}-\tilde{\omega}\right) e^{-i \Omega_{k} t}}{4\left(\Omega_{k}^{2}-\tilde{\omega}^{2}\right)^{2}+\pi^{2} g^{2} \Omega_{k}^{2}}\right]
\end{aligned}
$$




$$
\begin{aligned}
=g \sqrt{\frac{\omega_{k}}{\tilde{\omega}}} \sqrt{g \Delta \omega} \int_{-\infty}^{\infty} d^{\prime} \Omega \frac{\Omega(\Omega+\tilde{\omega}) e^{i \Omega t}}{\left(\Omega-\omega_{k}\right)\left[4\left(\Omega^{2}-\tilde{\omega}^{2}\right)^{2}+\pi^{2} g^{2} \Omega^{2}\right]} \\
-2 \frac{\sqrt{g \Delta \omega} \omega_{k}\left(\omega_{k}+\tilde{\omega}\right)\left(\tilde{\omega}^{2}-\omega_{k}^{2}\right) e^{i \omega_{k} t}}{\sqrt{\tilde{\omega} \omega_{k}}\left[4\left(\omega_{k}^{2}-\tilde{\omega}^{2}\right)^{2}+\pi^{2} g^{2} \omega_{k}^{2}\right]}
\end{aligned}
$$

where in passing to the second equality we have used the even symmetry of the integrand, factored the term $\left(\omega_{k}+\Omega\right)$, also we have used Eq. (B.12) to eliminate the singularity $\left(\Omega_{k}^{2}-\omega_{k}^{2}\right)^{-1}$ and finally we set $\Omega_{k}=\omega_{k}$. The prime in the integrand of Eq. (B.13) means that we are avoiding the points $\pm \omega_{k}$. Then when using the Cauchy theorem we have to multiply the residues in these poles by $i \pi$ instead of $2 i \pi$. Actually the pole at $-\omega_{k}$ has been factored. Then after using Cauchy theorem it is easy to obtain,

$$
\begin{aligned}
\alpha_{0 k}(t)= & \sqrt{\frac{\omega_{k}}{\tilde{\omega}}} \frac{\left(\tilde{\omega}+\omega_{k}\right)\left(2 \omega_{k}^{2}-2 \tilde{\omega}^{2}+i \pi g \omega_{k}\right)}{\left[4\left(\omega_{k}^{2}-\tilde{\omega}^{2}\right)^{2}+\pi^{2} g^{2} \omega_{k}^{2}\right]} \sqrt{g \Delta \omega} e^{i \omega_{k} t}+\sqrt{\frac{\omega_{k}}{\tilde{\omega}}} \frac{\sqrt{g \Delta \omega}}{4 \tilde{\tilde{\omega}}} \\
& \times\left[\frac{(4 \tilde{\tilde{\omega}}+4 \tilde{\omega}+i \pi g)}{\left(4 \tilde{\tilde{\omega}}-4 \omega_{k}+i \pi g\right)} e^{i \tilde{\tilde{\omega}} t}+\frac{(4 \tilde{\omega}-4 \tilde{\tilde{\omega}}+i \pi g)}{\left(4 \tilde{\tilde{\omega}}+4 \omega_{k}-i \pi g\right)} e^{-i \tilde{\tilde{\omega}} t}\right] e^{-\pi g t / 4} .(\mathrm{B} .
\end{aligned}
$$

Setting $\mu=k, \nu=0$ in Eq. (B.2) we obtain an expression for $\beta_{k 0}(t)$ that contains also the same singularity as in the case of $\alpha_{0 k}(t)$. Then, proceeding in the same way as in the above case we obtain

$$
\begin{aligned}
\beta_{k 0}(t)= & \sqrt{\frac{\omega_{k}}{\tilde{\omega}}} \frac{\left(\tilde{\omega}-\omega_{k}\right)\left(2 \omega_{k}^{2}-2 \tilde{\omega}^{2}+i \pi g \omega_{k}\right)}{\left[4\left(\omega_{k}^{2}-\tilde{\omega}^{2}\right)^{2}+\pi^{2} g^{2} \omega_{k}^{2}\right]} \sqrt{g \Delta \omega} e^{i \omega_{k} t}+\sqrt{\frac{\omega_{k}}{\tilde{\omega}}} \frac{\sqrt{g \Delta \omega}}{4 \tilde{\tilde{\omega}}} \\
& \times\left[\frac{(4 \tilde{\omega}-4 \tilde{\tilde{\omega}}-i \pi g)}{\left(4 \tilde{\tilde{\omega}}-4 \omega_{k}+i \pi g\right)} e^{i \tilde{\tilde{\omega}} t}+\frac{(4 \tilde{\omega}+4 \tilde{\tilde{\omega}}-i \pi g)}{\left(4 \tilde{\tilde{\omega}}+4 \omega_{k}-i \pi g\right)} e^{-i \tilde{\tilde{\omega}} t}\right] e^{-\pi g t / 4} . \text { B. }
\end{aligned}
$$

\section{References}

[1] J. S. Langer in Solids Far from Equilibrium, Ed. C. Godrèche (Cambridge University Press, Cambridge, 1992); D. J. Evans and G. P. Morriss, Statistical 
Mechanics of Non-Equilibrium Liquids (Academic Press, London, 1990).

[2] K. Rajagopal and F. Wilczek, Nucl. Phys. B 399, 395 (1995); ibid. B 404 (1993) 577.

[3] L. Kofman, A. Linde and A. A. Starobinskii, Phys. Rev. D 56 (1997) 3258.

[4] M. Gleiser and R. O. Ramos, Phys. Rev. D 50 (1994) 2441.

[5] A. Berera, M. Gleiser and R. O. Ramos, Phys. Rev. D 58 (1998) 123508.

[6] A. Berera and R. O. Ramos, Phys. Rev. D 63 (2001) 103509.

[7] D. G. Barci, E. S. Fraga and R. O. Ramos, Phys. Rev. Lett. 85 (2000) 479; Laser Phys. 12 (2002) 43; D. G. Barci, E. S. Fraga, M. Gleiser and R. O. Ramos, Physica A 317 (2003) 535.

[8] R. O. Ramos and F. A. R. Navarro, Phys. Rev. D 62 (2000) 085016.

[9] K. Chou, Z. Su, B. Hao and L. Yu, Phys. Rep. 118 (1985) 1; N. P. Landsman and Ch. G. van Weert, Phys. Rep. 145 (1987) 141.

[10] H. R. Lewis, Jr. and W. B. Riesenfeld, J. Math. Phys. 10 (1969) 1458.

[11] S. P. Kim and C. H. Lee, Phys. Rev. D 62 (2000) 125020.

[12] S. P. Kim, S. Sengupta and F. C. Khanna, Phys. Rev. D 64 (2001) 105026.

[13] P. G. Drazin and R. S. Johnson, Solitons: An Introduction (Cambridge University Press, Cambridge, England, 1993).

[14] I. S. Gradshteyn and L. M. Ryzhic, Table of Integrals Series and Products (Academic Press, Inc., New York, 1980).

[15] O. Éboli, R. Jackiw and S.-Y. Pi, Phys. Rev. D 37 (1988) 3557.

[16] M. Abramowitz and I. Stegun, Handbook of Mathematical Functions (Dover Publications, Inc., New York, 1964). 
[17] A. O. Caldeira and A. J. Leggett, Ann. Phys. (N.Y.) 149 (1983) 374.

[18] N. Andion, A. P. C. Malbouisson and A. Mattos Neto, J. Phys. A 34 (2001) 3735 .

[19] S. P. Kim and C. H. Lee, Phys. Rev. D 65 (2002) 045013. 


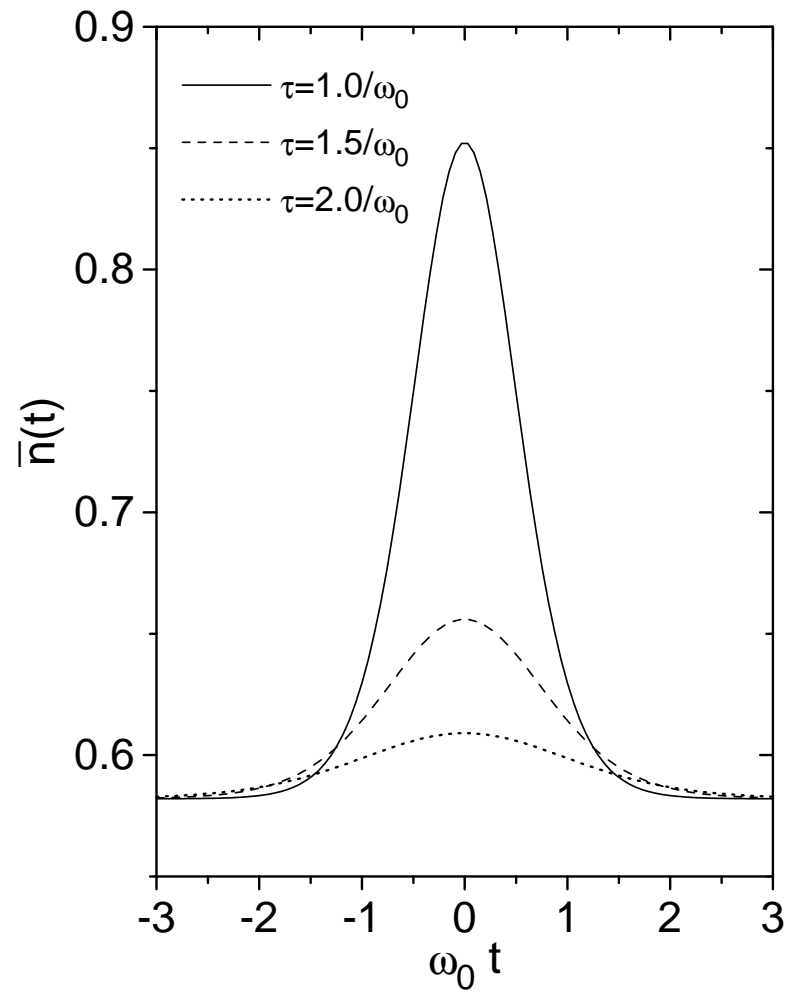

Fig. B.1. The number density for $\omega_{0} \beta=1$ and for decay times (in units of $1 / \omega_{0}$ ) $\tau=1.0,1.5$ and 2.0 . 


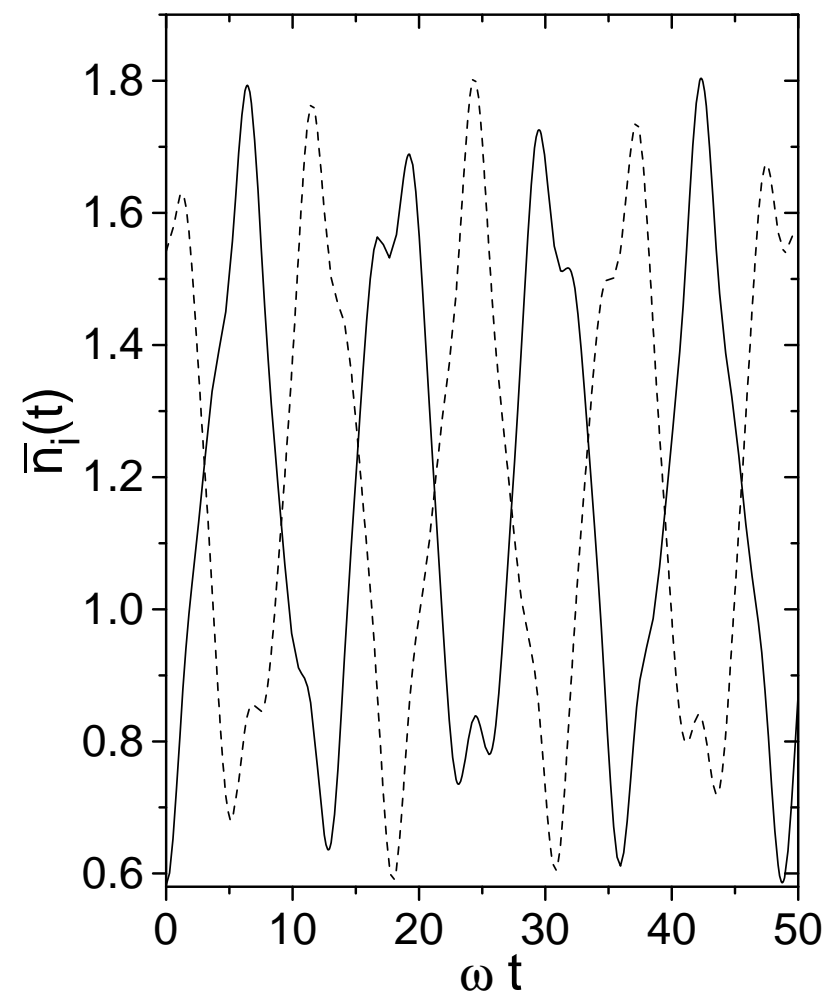

Fig. B.2. The time evolution for the number densities $\bar{n}_{1}(t)$ (dashed curve) and $\bar{n}_{2}(t)$ (continuous curve) for $\omega_{1}=\omega_{2}=\omega, \omega \beta_{1}=1 / 2, \omega \beta_{2}=1$ and $\lambda=\omega^{2} / 2$. 


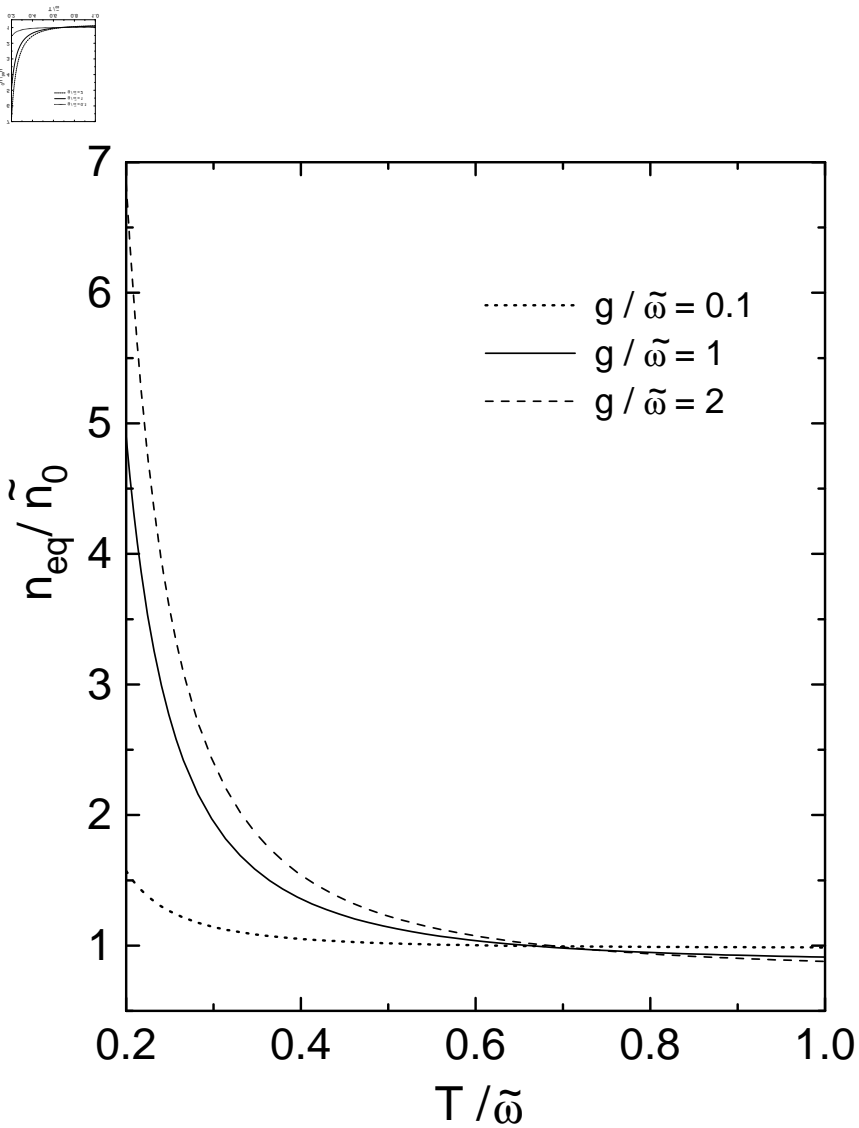

Fig. B.3. The system's equilibrium distribution, Eq. (130), normalized by the distribution $\tilde{n}_{0}=1 /[\exp (\beta \tilde{\omega})-1]$, as a function of the (scaled) temperature $T$ and for different values of the coupling constant $g$. 